\title{
Moment explosions in the rough Heston model
}

\author{
Stefan Gerhold ${ }^{1}$ (D) Christoph Gerstenecker $^{1} \cdot$ Arpad Pinter $^{1}$
}

Received: 24 July 2018 / Accepted: 19 August 2019 / Published online: 21 September 2019

(C) The Author(s) 2019

\begin{abstract}
We show that the moment explosion time in the rough Heston model, introduced by El Euch and Rosenbaum in 2016, is finite if and only if it is finite for the classical Heston model. Upper and lower bounds for the explosion time are established, as well as an algorithm to compute the explosion time (under some restrictions). We show that the critical moments are finite for all maturities. For negative correlation, we apply our algorithm for the moment explosion time to compute the lower critical moment.
\end{abstract}

Keywords Option pricing $\cdot$ Rough volatility $\cdot$ Rough Heston model $\cdot$ Moment explosion · Volterra integral equation

Mathematics Subject Classification $91 \mathrm{G} 20 \cdot 45 \mathrm{D} 05$

JEL Classification G13 · C65

\section{Introduction}

It has long been known that the marginal distributions of a realistic asset price model should not feature tails that are too thin (as, e.g., in the Black-Scholes model). In many models that have been proposed, the tails are of power law type. Consequently, not all moments of the asset price are finite Existence of the moments has been thoroughly investigated for classical models; in particular, we mention here Keller-Ressel's work (Keller-Ressel 2011) on affine stochastic volatility models. Precise information on the critical moments - the exponents where the stock price ceases to be integrable,

Financial support from the Austrian Science Fund (FWF) under Grants P 24880 and P 30750 is gratefully acknowledged.

\footnotetext{
Stefan Gerhold

sgerhold@fam.tuwien.ac.at

Christoph Gerstenecker

christoph.gerstenecker@fam.tuwien.ac.at

1 TU Wien, Vienna, Austria
} 
depending on maturity - is of interest for several reasons It allows to approximate the wing behavior of the volatility smile, to assess the convergence rate of some numerical procedures and to identify models that would assign infinite prices to certain financial products. We refer to Andersen and Piterbarg (2007), Keller-Ressel (2011) and the article Moment Explosions in Cont (2014) for further details and references on these motivations. Moreover, when using the Fourier representation to price options, choosing a good integration path (equivalently, a good damping parameter) to avoid highly oscillatory integrands requires knowing the strip of analyticity of the characteristic function. Its boundaries are described by the critical moments (Lee 2004b; Lord and Kahl 2007). See Sect. 10 for some details.

In recent years, attention has shifted in financial modeling from the classical (jump-)diffusion and Lévy models to rough volatility models. Since the pioneering work by Gatheral et al. (2018), the literature on these non-Markovian stochastic volatility models, inspired by fractional Brownian motion, has grown rapidly. We refer to Alòs et al. (2007), Comte et al. (2012), Comte and Renault (1998) and Fukasawa (2011) for earlier work on fractional volatility modeling, and, for example, to Bayer et al. (2017) for many further references. In the present paper, we provide some results on the explosion time and the critical moments of the rough Heston model. While there are several "rough" variants of the Heston model, we work with the one proposed by El Euch and Rosenbaum $(2018,2019)$. The dynamics of this model are given by

$$
\begin{aligned}
\mathrm{d} S_{t}= & S_{t} \sqrt{V_{t}} \mathrm{~d} W_{t}, \quad S_{0}>0, \\
V_{t}= & V_{0}+\frac{1}{\Gamma(\alpha)} \int_{0}^{t}(t-s)^{\alpha-1} \lambda\left(\bar{v}-V_{s}\right) \mathrm{d} s \\
& +\frac{1}{\Gamma(\alpha)} \int_{0}^{t}(t-s)^{\alpha-1} \xi \sqrt{V_{s}} \mathrm{~d} Z_{s}, \\
d\langle W, Z\rangle_{t}= & \rho \mathrm{d} t,
\end{aligned}
$$

where $W$ and $Z$ are correlated Brownian motions, $\rho \in(-1,1)$ and $\lambda, \xi, \bar{v}$ are positive parameters. The smoothness parameter $\alpha$ is in $\left(\frac{1}{2}, 1\right)$. (For $\alpha=1$, the model clearly reduces to the classical Heston model.) W.l.o.g. we assume zero interest rate. Besides having a microstructural foundation, this model features a characteristic function that can be evaluated numerically in an efficient way, by solving a fractional Riccati equation (equivalently, a nonlinear Volterra integral equation; see Sect. 2). Its tractability makes the rough Heston model attractive for practical implementation and at the same time facilitates our analysis.

We first analyze the explosion time, i.e., the maturity at which a fixed moment explodes. While the explosion time of the classical Heston model has an explicit formula, for rough Heston we arrive at a well-known hard problem: computing the explosion time of the solution of a nonlinear Volterra integral equation (VIE) of the second kind. There is no general algorithm known, and in most cases that have been studied in the literature, only bounds are available. See Roberts (1998) for an overview. Using the specific structure of our case, we show that the explosion time is finite if and only if it is finite for the classical Heston model, and we provide a lower and an upper bound (Sects. 3-5). As a by-product, the validity of the fractional Riccati equation, 
respectively, the VIE, for all moments (including complex exponents) is established in Sect. 6. In Sect. 7, we derive an algorithm to compute the explosion time, under some restrictions on the parameters.

The critical moments are finite for all maturities (Sect. 8) and can be computed by numerical root finding (Sect. 9). Our approach has two limitations: First, to compute the critical moments, maturity must not be too high. Second, our algorithm can compute the upper critical moment only for $\rho>0$ and the lower critical moment for $\rho<0$. As the latter is the more important case in practice, we focus on the left wing of implied volatility when recalling the relation between critical moments and strike asymptotics (Lee's moment formula; see Sect. 11).

Corollary 3.1 in El Euch and Rosenbaum (2018) is related to our results. For each maturity, it gives explicit lower and upper bounds for the critical moments. Inverting them yields a lower bound for the explosion time; the latter is not comparable to our bounds. Further bounds are contained in the recent preprint (Keller-Ressel and Majid 2019).

\section{Notation and preliminaries}

We assume in most of our analysis that $\alpha \in\left(\frac{1}{2}, 1\right)$. Some of the subsequent definitions and results are valid for larger intervals, but then the range of $\alpha$ is always explicitly stated. The letter $k(\cdot)$ will always denote the fractional convolution kernel $k(t-s)=$ $\frac{1}{\Gamma(\alpha)}(t-s)^{\alpha-1}$, unless explicitly stated otherwise.

El Euch and Rosenbaum (2019) established a semi-explicit representation of the moment generating function (mgf) of the $\log$-price $X_{t}=\log \left(S_{t} / S_{0}\right)$ in the rough Heston model. The mgf is given by

$$
\mathbb{E}\left[\mathrm{e}^{u X_{t}}\right]=\exp \left(\bar{v} \lambda I_{t}^{1} \psi(u, t)+v_{0} I_{t}^{1-\alpha} \psi(u, t)\right)
$$

where $\psi$ satisfies a fractional Riccati differential equation (see below). The constraint $\rho \in(-1 / \sqrt{2}, 1 / \sqrt{2}]$ from El Euch and Rosenbaum (2019) was removed recently in El Euch and Rosenbaum (2018). The paper (Alòs et al. 2017) contains an alternative derivation of the fractional Riccati equation, and Abi Jaber et al. (2017) has more general results, embedding the rough Heston model into the new class of affine Volterra processes. Recall the following definition [see e.g., Kilbas et al. (2006)]:

Definition 1 The (left-sided) Riemann-Liouville fractional integral $I_{t}^{\alpha}$ of order $\alpha \in$ $(0, \infty)$ of a function $f$ is given by

$$
I_{t}^{\alpha} f(t):=\frac{1}{\Gamma(\alpha)} \int_{0}^{t}(t-s)^{\alpha-1} f(s) \mathrm{d} s
$$

whenever the integral exists, and the (left-sided) Riemann-Liouville fractional derivative $D_{t}^{\alpha}$ of order $\alpha \in[0,1)$ of $f$ is given by

$$
D_{t}^{\alpha} f(t):=\frac{\mathrm{d}}{\mathrm{d} t} I^{1-\alpha} f(t)
$$


whenever this expression exists.

(The fractional derivative $D_{t}^{\alpha}$ can be defined for $\alpha>1$ as well, but this is not needed in our context.) The function $\psi$ from (1) is the unique continuous solution of the fractional Riccati initial value problem

$$
\begin{aligned}
D_{t}^{\alpha} \psi(u, t) & =R(u, \psi(u, t)), \\
I_{t}^{1-\alpha} \psi(u, 0) & =0,
\end{aligned}
$$

where $R$ is defined as

$$
R(u, w)=c_{1}(u)+c_{2}(u) w+c_{3} w^{2},
$$

with coefficients

$$
\begin{aligned}
c_{1}(u) & =\frac{1}{2} u(u-1), \\
c_{2}(u) & =\rho \xi u-\lambda, \\
c_{3} & =\frac{1}{2} \xi^{2}>0 .
\end{aligned}
$$

For $\alpha=1$, this becomes a standard Riccati differential equation, which admits a wellknown explicit solution (Gatheral 2006, Chapter 2). The roots of $R(u, \cdot)$ are located at the points $\frac{1}{c_{3}}\left(-e_{0}(u) \pm \sqrt{e_{1}(u)}\right)$ with

$$
\begin{aligned}
& e_{0}(u):=\frac{1}{2} c_{2}(u)=\frac{1}{2}(\rho \xi u-\lambda), \\
& e_{1}(u):=e_{0}(u)^{2}-c_{3} c_{1}(u)=e_{0}(u)^{2}-\frac{1}{4} \xi^{2} u(u-1) .
\end{aligned}
$$

The following result, relating fractional differential equations and Volterra integral equations, is a special case of Theorem 3.10 in Kilbas et al. (2006).

Theorem 1 Let $\alpha \in(0,1), T>0$ and suppose that $\psi \in C[0, T]$ and $H \in C(\mathbb{R})$. Then, $\psi$ satisfies the fractional differential equation

$$
\begin{aligned}
D_{t}^{\alpha} \psi(t) & =H(\psi(t)), \\
I_{t}^{1-\alpha} \psi(0) & =0
\end{aligned}
$$

if and only if it satisfies the Volterra integral equation (VIE)

$$
\psi(t)=\frac{1}{\Gamma(\alpha)} \int_{0}^{t}(t-s)^{\alpha-1} H(\psi(s)) \mathrm{d} s .
$$

Using Theorem 1, the Riccati differential equation (4) with initial value (5) can be transformed into the nonlinear Volterra integral equation

$$
\psi(u, t)=\frac{1}{\Gamma(\alpha)} \int_{0}^{t}(t-s)^{\alpha-1} R(u, \psi(u, s)) \mathrm{d} s .
$$


This integral equation was used in El Euch and Rosenbaum (2019) to compute $\psi$ numerically. The function

$$
f(u, t):=c_{3} \psi(u, t)
$$

solves

$$
f(u, t)=\frac{1}{\Gamma(\alpha)} \int_{0}^{t}(t-s)^{\alpha-1} G(u, f(u, s)) \mathrm{d} s
$$

with nonlinearity

$$
\begin{aligned}
G(u, w) & =c_{3} R\left(u, w / c_{3}\right) \\
& =\left(w+e_{0}(u)\right)^{2}-e_{1}(u),
\end{aligned}
$$

where $e_{0}(u)$ and $e_{1}(u)$ are defined in (7) and (8). Equation (9) is a nonlinear Volterra integral equation with weakly singular kernel; it will be used to analyze the blowup behavior of $f$ (and thus of $\psi$ ) in the following sections. We quote the following standard existence and uniqueness result for equations of this kind.

Theorem 2 Let $\alpha \in(0,1), g \in C[0, \infty)$, and suppose that $H: \mathbb{R} \rightarrow \mathbb{R}$ is locally Lipschitz continuous. Then, there is $T^{*} \in(0, \infty]$ such that the Volterra integral equation

$$
\psi(t)=g(t)+\int_{0}^{t}(t-s)^{\alpha-1} H(\psi(s)) \mathrm{d} s
$$

has a unique continuous solution $\psi$ on $\left[0, T^{*}\right)$, and there is no continuous solution on any larger right-open interval $\left[0, T^{* *}\right)$.

Proof For existence and uniqueness on a sufficiently small interval $\left[0, T_{0}\right]$ with $T_{0}>0$, see, for example, Theorem 3.1.4 in Brunner's recent monograph (Brunner 2017). The continuation to a maximal right-open interval is discussed there as well [p. 107; see also Sect. 12 of Gripenberg et al. (1990)].

In El Euch and Rosenbaum (2019), the fractional Riccati equation was established for $u \in i \mathbb{R}$, whereas we are interested in $u \in \mathbb{R}$. The justification of (4)-(5), and thus of (9), for $u \in \mathbb{R}$ hinges on a result from El Euch and Rosenbaum (2018) and the analytic dependence of $f(u, t)$ on $u$. See Sects. 5 and 6 for details.

We write

$$
T_{\alpha}^{*}(u):=\sup \left\{t \geq 0: \mathbb{E}\left[S_{t}^{u}\right]<\infty\right\}, \quad u \in \mathbb{R},
$$

for the moment explosion time in the rough Heston model. In the classical case $(\alpha=1)$, the explicit expression

$$
T_{1}^{*}(u)= \begin{cases}\int_{0}^{\infty} \frac{1}{R(u, w)} \mathrm{d} w, & R(u, \cdot) \text { has no roots on }[0, \infty), \\ \infty, & \text { otherwise }\end{cases}
$$




$$
= \begin{cases}\frac{1}{\sqrt{\left|e_{1}(u)\right|}}\left(\frac{\pi}{2}-\arctan \left(\frac{e_{0}(u)}{\sqrt{\left|e_{1}(u)\right|}}\right)\right), & e_{1}(u)<0, \\ \frac{1}{2 \sqrt{e_{1}(u)}} \log \left(\frac{e_{0}(u)+\sqrt{e_{1}(u)}}{e_{0}(u)-\sqrt{e_{1}(u)}}\right), & e_{1}(u) \geq 0, e_{0}(u)>0, \\ \infty, & e_{1}(u) \geq 0, e_{0}(u)<0,\end{cases}
$$

has been found by Andersen and Piterbarg (2007) (see also Keller-Ressel 2011). It is a consequence of the explicit characteristic function, which is not available for the rough Heston model. We distinguish between the following cases for $u \in \mathbb{R}$ :

(A) $c_{1}(u)>0, e_{0}(u) \geq 0$,

(B) $c_{1}(u)>0, e_{0}(u)<0$ and $e_{1}(u)<0$,

(C) $c_{1}(u)>0, e_{0}(u)<0$ and $e_{1}(u) \geq 0$,

(D) $c_{1}(u) \leq 0$.

As several of our results deal with $\rho<0$ and $u$ satisfying case (A), we explicitly note

$$
\text { case (A) for } \rho<0: \quad u \leq \frac{\lambda}{\rho \xi} \text {. }
$$

Note that cases (A) and (B) combined are exactly the cases in which the moment explosion time $T_{1}^{*}(u)$ in the classical Heston model is finite, by (13). We can now state our first main result.

Theorem 3 For $u \in \mathbb{R}$, the moment explosion time $T_{\alpha}^{*}(u)$ of the rough Heston model is finite if and only if $u$ satisfies $(A)$ or $(B)$. This is equivalent to $T_{1}^{*}(u)$ (explosion time of the classical Heston model) being finite.

The proof of Theorem 3 consists of two main parts. First, Propositions 2-5 discuss the blowup behavior of the solution of (9) in cases (A)-(D), and Lemma 3 in Sect. 4 shows that blowup of $f$ leads to blowup of the right-hand side of (1). Second, we show in Sect. 5 that the explosion time of $f(u, \cdot)$ [the solution of (9)] agrees with $T_{\alpha}^{*}(u)$ (the explosion time of the rough Heston model) for all $u \in \mathbb{R}$. As mentioned after Theorem 2, this is not obvious from the results in the existing literature.

\section{Explosion time of the Volterra integral equation}

We begin by citing a result from Brunner and Yang (2012) which characterizes the blowup behavior of nonlinear Volterra integral equations defined by positive and increasing functions. "Blowup in finite time" means that there is a finite number $\hat{T}>0$ such that $h$ is defined on $[0, \hat{T})$ and satisfies $\lim _{t \uparrow \hat{T}}|h(t)|=\infty$. We note that some arguments in our subsequent proofs (from Proposition 2 onward) are similar to arguments used in Brunner and Yang (2012). Alternatively, it should be possible to extend the arguments in Appendix A of Gatheral and Keller-Ressel (2019); there, $u$ is in $[0,1]$.

Proposition 1 Assume that $H:[0, \infty) \rightarrow[0, \infty)$ is continuous and the following conditions hold: 
(H1) $H(0)=0$ and $H$ is strictly increasing,

(H2) $\lim _{w \rightarrow \infty} H(w) / w=\infty$,

(P) $\phi:[0, \infty) \rightarrow(0, \infty)$ is a positive, nondecreasing, continuous function,

(K) $k:(0, \infty) \rightarrow[0, \infty)$ is locally integrable and $K(t):=\int_{0}^{t} k(z) \mathrm{d} z>0$ is a nondecreasing function.

Furthermore, assume $\lim _{t \rightarrow \infty} \phi(t)=\infty$ and $k(z)=c z^{\alpha-1}$ with $\alpha>0$ and $c>0$. Then, the solution $h$ of the Volterra integral equation

$$
h(t)=\phi(t)+\int_{0}^{t} k(t-s) H(h(s)) \mathrm{d} s
$$

blows up in finite time if and only if

$$
\int_{U}^{\infty}\left(\frac{w}{H(w)}\right)^{1 / \alpha} \frac{\mathrm{d} w}{w}<\infty
$$

for all $U>0$.

Proof This is a special case of Corollary 2.22 in Brunner and Yang (2012), with $H$ (in their notation, $G$ ) not depending on time.

In case (A), all assumptions of Proposition 1 are satisfied and only the integrability condition (14) has to be checked to determine whether the solution $f$ of (9) blows up in finite time.

Proposition 2 In case (A), the solution $f$ of (9) starts at 0 , is positive thereafter and blows up in finite time.

Proof Fix $u \in \mathbb{R}$ such that $c_{1}(u)>0$ and $e_{0}(u) \geq 0$. Note that $e_{0}^{2}-e_{1}>0$ in this case. (Here and in the following, we will often suppress $u$ in the notation.) If we write the Volterra integral equation (9) in the form

$$
f(t)=\phi(t)+\int_{0}^{t} k(t-s) \bar{G}(f(s)) \mathrm{d} s
$$

with kernel $k(t-s)=(t-s)^{\alpha-1} / \Gamma(\alpha)$, nonlinearity $\bar{G}(w)=w^{2}+2 e_{0} w$ and $\phi(t)=$ $\frac{e_{0}^{2}-e_{1}}{\Gamma(1+\alpha)} t^{\alpha}$, using (10) and (44), then the conditions $c_{1}>0$ and $e_{0} \geq 0$ guarantee that $\phi$ and $\bar{G}$ are positive and strictly increasing on $(0, \infty)$ with $\phi(0)=\bar{G}(0)=0$. Hence, the solution $f$ is positive for positive values, and $f(0)=0$. (Positivity follows from Lemma 2.4 in Brunner and Yang (2012), or from Lemma 3.2.11 in Brunner (2017).) It is easy to check that all the assumptions $(\mathrm{H} 1),(\mathrm{H} 2),(\mathrm{P})$ and $(\mathrm{K})$ of Proposition 1 are satisfied. Moreover, $\lim _{t \rightarrow \infty} \phi(t)=\infty$ and

$$
\int_{U}^{\infty}\left(\frac{w}{\bar{G}(w)}\right)^{1 / \alpha} \frac{\mathrm{d} w}{w} \leq \int_{U}^{\infty} w^{-1-1 / \alpha} \mathrm{d} w<\infty
$$

for all $U>0$. By Proposition 1, the solution $f$ blows up in finite time. 
In case (B), Proposition 1 cannot be applied directly to the solution $f$ of (9). Hence, the Volterra integral equation has to be modified in order to satisfy the assumptions of Proposition 1 in a way that $f$ is still a subsolution of the modified equation, i.e., $f$ satisfies (9) with " $\geq$ " instead of "=." First, we provide a comparison lemma for solutions and subsolutions.

Lemma 1 Let $H:[0, \infty) \rightarrow(0, \infty)$ be a strictly increasing, continuous function and $T>0$. Suppose that $g$ is the unique continuous solution of the Volterra integral equation

$$
g(t)=\int_{0}^{t} k(t-s) H(g(s)) \mathrm{d} s, \quad t \in[0, T]
$$

where $k$ satisfies condition $(K)$ from Proposition 1 . If $f$ is a continuous subsolution,

$$
f(t) \geq \int_{0}^{t} k(t-s) H(f(s)) \mathrm{d} s, \quad t \in[0, T],
$$

then $f(t) \geq g(t)$ holds for all $t \in[0, T]$.

Proof For any $c \in(0, T)$, define $f_{c}(t):=f(t+c)$ for $t \in[0, T-c]$. From the positivity of $H$, it follows that $f_{c}(0)=f(c)>0$ and

$$
f_{c}(t) \geq \int_{0}^{t} k(t-s) H\left(f_{c}(s)\right) \mathrm{d} s, \quad t \in[0, T-c] .
$$

Since $g(0)=0$, it follows that $g(0)<f_{c}(0)$. We want to show $g<f_{c}$ on the whole interval $[0, T-c]$. Therefore, suppose that $t \in(0, T-c]$ exists such that $0 \leq g(s)<f_{c}(s)$ for all $s \in[0, t)$ and $g(t)=f_{c}(t)$. However, because $H$ is strictly increasing, we have

$$
0=f_{c}(t)-g(t) \geq \int_{0}^{t} k(t-s)\left(H\left(f_{c}(s)\right)-H(g(s))\right) \mathrm{d} s>0,
$$

which is a contradiction. Hence, the inequality $g(t)<f_{c}(t)=f(t+c)$ holds for all $t \in[0, T-c]$. Since $c \in(0, T)$ was arbitrary, the result follows easily.

In what follows, we will not consider other kernels than

$$
k(t-s)=\frac{1}{\Gamma(\alpha)}(t-s)^{\alpha-1},
$$

and so $k(\cdot)$ always has this meaning in the rest of the paper.

Proposition 3 In case $(B)$, the solution $f$ of (9) starts at 0 , is positive thereafter and blows up in finite time. 
Proof Fix $u \in \mathbb{R}$ such that $c_{1}(u)>0, e_{0}(u)<0$ and $e_{1}(u)<0$. Note that in this case, the nonlinearity $G$ is obviously positive by (10). However, $G$ is strictly decreasing on $\left[0,-e_{0}\right]$. To deal with this problem, let $0<a<-e_{1}$ and define the modified nonlinearity $\bar{G}_{a}$ as

$$
\bar{G}_{a}(w)= \begin{cases}w \frac{a+e_{1}}{e_{0}}+a, & 0 \leq w<-e_{0} \\ G(w), & w \geq-e_{0}\end{cases}
$$

Then, $\bar{G}_{a}$ is a positive, strictly increasing, Lipschitz continuous function that starts at $a$ and $\bar{G}_{a} \leq G$. Let $\bar{f}$ be the unique continuous solution (recall Theorem 2) of the Volterra integral equation

$$
\bar{f}(t)=\int_{0}^{t} k(t-s) \bar{G}_{a}(\bar{f}(s)) \mathrm{d} s=\phi(t)+\int_{0}^{t} k(t-s) \bar{G}(\bar{f}(s)) \mathrm{d} s
$$

with $k(t-s)=(t-s)^{\alpha-1} / \Gamma(\alpha), \bar{G}=\bar{G}_{a}-a$ and $\phi(t)=\frac{a}{\Gamma(1+\alpha)} t^{\alpha}$. Note that the second equality in (16) follows from (44). Due to the positivity of $\phi$ and $\bar{G}$ on $(0, \infty)$, the solution $\bar{f}$ is positive on $(0, \infty)$ as well. The functions $\phi, \bar{G}$ and $k$ satisfy the assumptions (H1), (H2), (P) and (K) in Proposition 1. Furthermore, $\lim _{t \rightarrow \infty} \phi(t)=\infty$ and $\bar{G}$ satisfies (14). By Proposition $1, \bar{f}$ blows up in finite time. Because $f$ satisfies (9) and $\bar{G}_{a} \leq G$, it follows that $f$ is a subsolution of the modified Volterra integral equation, i.e.,

$$
f(t) \geq \int_{0}^{t} k(t-s) \bar{G}_{a}(f(s)) \mathrm{d} s .
$$

Now, Lemma 1 implies $f \geq \bar{f}$. Consequently, $f$ blows up as well.

Cases (C) and (D) are the cases where the solution $f$ of (9) does not blow up in finite time. In fact, $f$ does not blow up at all, as we will see. The following lemma provides the key argument for both cases.

Lemma 2 Let $H:[0, \infty) \rightarrow[0, \infty)$ be a Lipschitz continuous function that is positive on $[0, a)$ and satisfies $H \equiv 0$ on $[a, \infty)$ for an $a>0$. Then, the unique continuous solution $f$ of the Volterra integral equation

$$
f(t)=\int_{0}^{t} k(t-s) H(f(s)) \mathrm{d} s,
$$

where $k(t-s)=(t-s)^{\alpha-1} / \Gamma(\alpha)$, satisfies $0 \leq f(t) \leq$ a for all $t \geq 0$.

Proof The nonnegativity of $H$ implies $f \geq 0$. Suppose $t>0$ exists such that $f(t)>a$. By the continuity of $f$, there exists $0<t_{0}<t$ that satisfies $f\left(t_{0}\right)=a$ and $f(s)>a$ for all $s \in\left(t_{0}, t\right)$. From $H \equiv 0$ on $[a, \infty)$, we have

$$
\int_{t_{0}}^{t} k(t-s) H(f(s)) \mathrm{d} s=0 .
$$


Since $H$ is nonnegative and $k$ is decreasing,

$$
\begin{aligned}
0 & <f(t)-f\left(t_{0}\right) \\
& =\int_{t_{0}}^{t} k(t-s) H(f(s)) \mathrm{d} s+\int_{0}^{t_{0}}\left(k(t-s)-k\left(t_{0}-s\right)\right) H(f(s)) \mathrm{d} s \\
& =\int_{0}^{t_{0}}\left(k(t-s)-k\left(t_{0}-s\right)\right) H(f(s)) \mathrm{d} s \leq 0,
\end{aligned}
$$

which is a contradiction. Therefore, $f$ satisfies $0 \leq f(t) \leq a$ for all $t \geq 0$.

Proposition 4 In case $(C)$, the solution $f$ of (9) is nonnegative and bounded and exists globally.

Proof Fix $u \in \mathbb{R}$ such that $c_{1}(u)>0, e_{0}(u)<0$ and $e_{1}(u) \geq 0$. Note that the inequality $0 \leq e_{1}=e_{0}^{2}-c_{1} c_{3}<e_{0}^{2}$ implies $a:=-e_{0}-\sqrt{e_{1}}>0$. Moreover, from (10), it follows that $a$ is the smallest positive root of $G$. Define the nonlinearity $\bar{G}$ as

$$
\bar{G}(w):= \begin{cases}G(w), & 0 \leq w \leq a, \\ 0, & w>a .\end{cases}
$$

Then, $\bar{G}$ is a nonnegative, Lipschitz continuous function that starts at $e_{0}^{2}-e_{1}>0$. Therefore, Lemma 2 yields that the unique continuous solution $\bar{f}$ of

$$
\bar{f}(t)=\int_{0}^{t} k(t-s) \bar{G}(\bar{f}(s)) \mathrm{d} s
$$

is bounded with $0 \leq \bar{f}(t) \leq a$ for all $t \geq 0$. Since $\bar{G}=G$ on $[0, a]$, the function $\bar{f}$ solves the original Volterra integral equation

$$
\bar{f}(t)=\int_{0}^{t} k(t-s) G(\bar{f}(s)) \mathrm{d} s,
$$

and from the uniqueness of the solution, we obtain $f=\bar{f}$.

Proposition 5 In case (D), the solution $f$ of (9) is nonpositive and bounded and exists globally.

Proof Fix $u \in \mathbb{R}$ such that $c_{1}(u) \leq 0$, which is equivalent to $u \in[0,1]$. Note that $e_{1}=e_{0}^{2}-c_{1} c_{3}>e_{0}^{2}>0$ implies $a:=\sqrt{e_{1}}-e_{0}>0$. Moreover, from (10), it follows that $a$ is the smallest positive root of $G$. Define $f_{-}:=-f$, which satisfies

$$
f_{-}(t)=-\int_{0}^{t} k(t-s) G\left(-f_{-}(s)\right) \mathrm{d} s .
$$

If we define the nonlinearity $\bar{G}$ as

$$
\bar{G}(w):= \begin{cases}-G(-w), & 0 \leq w \leq a, \\ 0, & w>a\end{cases}
$$


then $\bar{G}$ is a nonnegative, Lipschitz continuous function that starts at $e_{1}-e_{0}^{2}>0$. With Lemma 2, we obtain that the unique continuous solution $\bar{f}$ of

$$
\bar{f}(t)=\int_{0}^{t} k(t-s) \bar{G}(\bar{f}(s)) \mathrm{d} s
$$

is bounded with $0 \leq \bar{f}(t) \leq a$ for all $t \geq 0$. Furthermore, $\bar{f}$ solves (17) because $\bar{G}(w)=-G(-w)$ for all $w \in[0, a]$. The uniqueness of the solution yields

$$
\bar{f}=f_{-}=-f .
$$

Hence, the solution $f$ is bounded with $-a \leq f(t) \leq 0$.

We have shown that (A) and (B) are exactly the cases in which the solution $f$ of the Volterra integral equation (9), and thus the solution $\psi$ of the fractional Riccati differential equation (4) with initial value (5), blows up in finite time.

\section{Bounds for the explosion time}

We denote by $\hat{T}_{\alpha}(u)$ the explosion time of the solution $f(u, \cdot)$ of (9). We now establish lower and upper bounds for $\hat{T}_{\alpha}(u)$, valid whenever it is finite [cases (A) and (B)]. As we will see later, $\hat{T}_{\alpha}(u)$ agrees with $T_{\alpha}^{*}(u)$, and so both bounds of this section hold for the explosion time $T_{\alpha}^{*}(u)$ of the rough Heston model.

Theorem 4 In cases $(A)$ and $(B)$, the blowup time $\hat{T}_{\alpha}(u)$ of the solution $f(u, \cdot)$ of $(9)$ satisfies

$$
\hat{T}_{\alpha}(u) \geq \Gamma(1+\alpha)^{1 / \alpha} \max _{r>1} \frac{\left(r^{\alpha}-1\right)^{1 / \alpha}}{r(r-1)} \int_{a(u)}^{\infty}\left(\frac{w}{G(u, w)}\right)^{1 / \alpha} \frac{\mathrm{d} w}{w},
$$

where $a(u)=0$ in case $(A)$ and $a(u)=-e_{0}(u)>0$ in case $(B)$.

Proof Fix $u$ satisfying the requirements of case (A) or (B). It follows from Propositions 2 and 3 that in either case the solution $f$ is nonnegative, starts at 0 and $\lim _{t \uparrow \hat{T}_{\alpha}} f(t)=\infty$. For any $n \in \mathrm{N}_{0}$, choose

$$
t_{n}:=\min \left\{t>0: f(t)=\left(c r^{n}\right)^{\alpha}+a\right\}
$$

with $r>1$ and $c>0$. Using the inequality $k\left(t_{n}-s\right)<k\left(t_{n-1}-s\right)$ for $s \in\left(0, t_{n-1}\right)$, the nonnegativity of $G$ and that $G$ is strictly increasing on $[a, \infty)$, we have for $n \in \mathrm{N}$

$$
\begin{aligned}
f\left(t_{n}\right) & =\int_{0}^{t_{n-1}} k\left(t_{n}-s\right) G(f(s)) \mathrm{d} s+\int_{t_{n-1}}^{t_{n}} k\left(t_{n}-s\right) G(f(s)) \mathrm{d} s \\
& \leq f\left(t_{n-1}\right)+G\left(f\left(t_{n}\right)\right) \int_{t_{n-1}}^{t_{n}} k\left(t_{n}-s\right) \mathrm{d} s
\end{aligned}
$$




$$
=f\left(t_{n-1}\right)+\frac{1}{\Gamma(1+\alpha)} G\left(f\left(t_{n}\right)\right)\left(t_{n}-t_{n-1}\right)^{\alpha} .
$$

Thus, we obtain for $n \in \mathrm{N}$

$$
\begin{aligned}
t_{n}-t_{n-1} & \geq \Gamma(1+\alpha)^{1 / \alpha}\left(\frac{f\left(t_{n}\right)-f\left(t_{n-1}\right)}{G\left(f\left(t_{n}\right)\right)}\right)^{1 / \alpha} \\
& =\Gamma(1+\alpha)^{1 / \alpha}\left(r^{\alpha}-1\right)^{1 / \alpha} \frac{c r^{n-1}}{G\left(\left(c r^{n}\right)^{\alpha}+a\right)^{1 / \alpha}} \\
& =\Gamma(1+\alpha)^{1 / \alpha} \frac{\left(r^{\alpha}-1\right)^{1 / \alpha}}{r(r-1)} \cdot \frac{c r^{n+1}-c r^{n}}{G\left(\left(c r^{n}\right)^{\alpha}+a\right)^{1 / \alpha}} \\
& \geq \Gamma(1+\alpha)^{1 / \alpha} \frac{\left(r^{\alpha}-1\right)^{1 / \alpha}}{r(r-1)} \int_{c r^{n}}^{c r^{n+1}}\left(\frac{1}{G\left(s^{\alpha}+a\right)}\right)^{1 / \alpha} \mathrm{d} s .
\end{aligned}
$$

Finally,

$$
\begin{aligned}
\hat{T}_{\alpha} & =t_{0}+\sum_{n=1}^{\infty}\left(t_{n}-t_{n-1}\right) \\
& \geq \Gamma(1+\alpha)^{1 / \alpha} \frac{\left(r^{\alpha}-1\right)^{1 / \alpha}}{r(r-1)} \int_{c r}^{\infty}\left(\frac{1}{G\left(s^{\alpha}+a\right)}\right)^{1 / \alpha} \mathrm{d} s .
\end{aligned}
$$

Maximization over $c>0$, then over $r>1$ and the substitution $w=s^{\alpha}+a$ yield the inequality (18).

For $\alpha \uparrow 1$, the right-hand side of (18) simplifies to

$$
\int_{a(u)}^{\infty} \frac{1}{G(u, x)} \mathrm{d} x=\int_{a(u) / c_{3}}^{\infty} \frac{1}{R(u, x)} \mathrm{d} x .
$$

In case (A), the lower bound (18) is sharp in the limit $\alpha \uparrow 1$ : We have $a(u)=0$ then, and therefore (20) is exactly the moment explosion time (12) of the classical Heston model.

Another lower bound for $\hat{T}_{\alpha}$ can be obtained from Corollary 3.1 in El Euch and Rosenbaum (2018). Numerical examples show that it is not comparable to the bound from our Theorem 4.

Theorem 5 In cases $(A)$ and $(B)$, the blowup time $\hat{T}_{\alpha}(u)$ of the solution $f(u, \cdot)$ of (9) satisfies

$$
\hat{T}_{\alpha}(u) \leq 4 \Gamma(1+\alpha)^{1 / \alpha} \int_{0}^{\infty}\left(\frac{w}{\hat{G}(u, w)}\right)^{1 / \alpha} \frac{\mathrm{d} w}{w},
$$

where $\hat{G}=G$ in case $(A)$, and $\hat{G} \equiv-e_{1}$ on $\left[0,-e_{0}\right)$ and $\hat{G}=G$ on $\left[-e_{0}, \infty\right)$ in case (B).

Proof Fix $u$ satisfying the requirements of case (A) or (B). From Propositions 2 and 3 , in either case the solution $f$ is positive on $(0, \infty)$, starts at 0 and $\lim _{t \uparrow \hat{T}_{\alpha}} f(t)=\infty$. 
For any $n \in \mathrm{N}_{0}$, choose

$$
t_{n}:=\max \left\{t<\hat{T}_{\alpha}: f(t)=\left(c r^{n}\right)^{\alpha}\right\}
$$

with $r>1$ and $c>0$. Define $\bar{G}:=G$ in case (A) and $\bar{G}:=\bar{G}_{a}$ from (15) in case (B). Since $\bar{G} \leq G$ and $\bar{G}$ is positive and strictly increasing, we have for $n \in \mathrm{N}$

$$
\begin{aligned}
f\left(t_{n}\right) & \geq \int_{0}^{t_{n}} k\left(t_{n}-s\right) \bar{G}(f(s)) \mathrm{d} s \\
& \geq \int_{t_{n-1}}^{t_{n}} k\left(t_{n}-s\right) \bar{G}(f(s)) \mathrm{d} s \\
& \geq \frac{1}{\Gamma(1+\alpha)} \bar{G}\left(f\left(t_{n-1}\right)\right)\left(t_{n}-t_{n-1}\right)^{\alpha} .
\end{aligned}
$$

Thus, we obtain for $n \in \mathrm{N}$

$$
\begin{aligned}
t_{n}-t_{n-1} & \leq \Gamma(1+\alpha)^{1 / \alpha}\left(\frac{f\left(t_{n}\right)}{\bar{G}\left(f\left(t_{n-1}\right)\right)}\right)^{1 / \alpha} \\
& =\Gamma(1+\alpha)^{1 / \alpha} \frac{c r^{n}}{\bar{G}\left(\left(c r^{n-1}\right)^{\alpha}\right)^{1 / \alpha}} \\
& =\Gamma(1+\alpha)^{1 / \alpha} \frac{r^{2}}{r-1} \cdot \frac{c r^{n-1}-c r^{n-2}}{\bar{G}\left(\left(c r^{n-1}\right)^{\alpha}\right)^{1 / \alpha}} \\
& \leq \Gamma(1+\alpha)^{1 / \alpha} \frac{r^{2}}{r-1} \int_{c r^{n-2}}^{c r^{n-1}}\left(\frac{1}{\bar{G}\left(s^{\alpha}\right)}\right)^{1 / \alpha} \mathrm{d} s .
\end{aligned}
$$

Therefore,

$$
\begin{aligned}
\hat{T}_{\alpha} & =t_{0}+\sum_{n=1}^{\infty}\left(t_{n}-t_{n-1}\right) \\
& \leq t_{0}+\Gamma(1+\alpha)^{1 / \alpha} \frac{r^{2}}{r-1} \int_{c r^{-1}}^{\infty}\left(\frac{1}{\bar{G}\left(s^{\alpha}\right)}\right)^{1 / \alpha} \mathrm{d} s .
\end{aligned}
$$

Note that from the definition of $t_{0}$, it depends on $c>0$ and $r>1$. The fact that $f$ is only zero at $t=0$ implies that $t_{0} \rightarrow 0$ as $c \downarrow 0$. Taking the limit $c \downarrow 0$, then minimizing over $r>1$ and substituting $w=s^{\alpha}$ yield

$$
\hat{T}_{\alpha} \leq 4 \Gamma(1+\alpha)^{1 / \alpha} \int_{0}^{\infty}\left(\frac{w}{\bar{G}(w)}\right)^{1 / \alpha} \frac{\mathrm{d} w}{w}
$$

In case (A), we are finished. In case (B), we have $\bar{G}=\bar{G}_{a}$. Then, the dominated convergence theorem for $a \uparrow-e_{1}$ yields the inequality (21). 


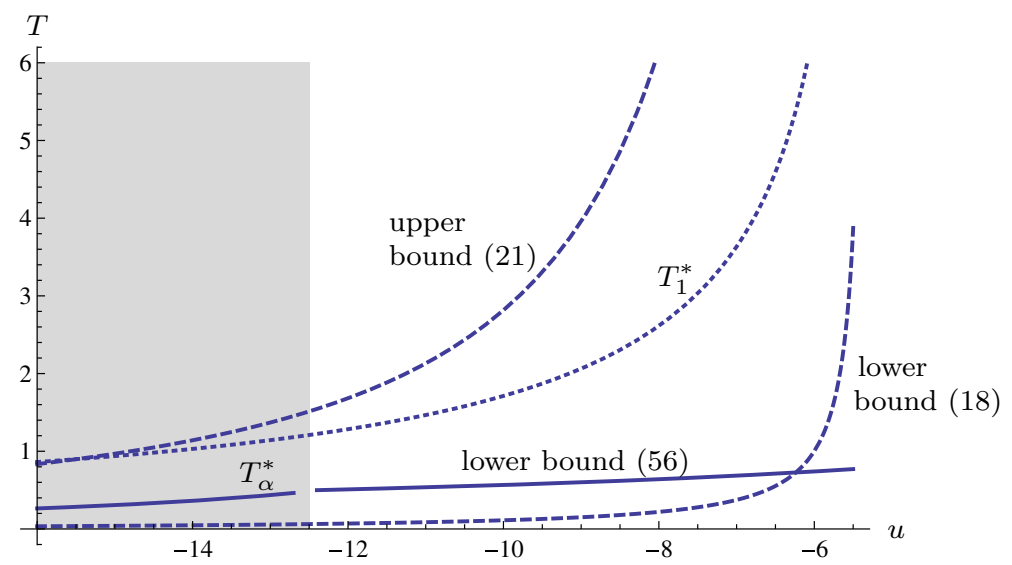

Fig. 1 Moment explosion time and bounds for $u<0$. The parameters are $\alpha=0.6, \rho=-0.8, \lambda=2$, and $\xi=0.2$. The gray rectangle sits above the interval $(-\infty, \lambda /(\xi \rho)]=(-\infty,-12.5]$ corresponding to case (A). Left solid curve: $T_{\alpha}^{*}$, computed by Algorithm 1. Right solid curve: lower bound, computed by Algorithm 2. Dashed curves: bounds from Theorems 4 and 5. Dotted curve: $T_{1}^{*}$ (classical Heston model). The explosion time $T_{\alpha}^{*}$ becomes infinite at $u \approx-5.29$, the smaller root of $e_{1}$

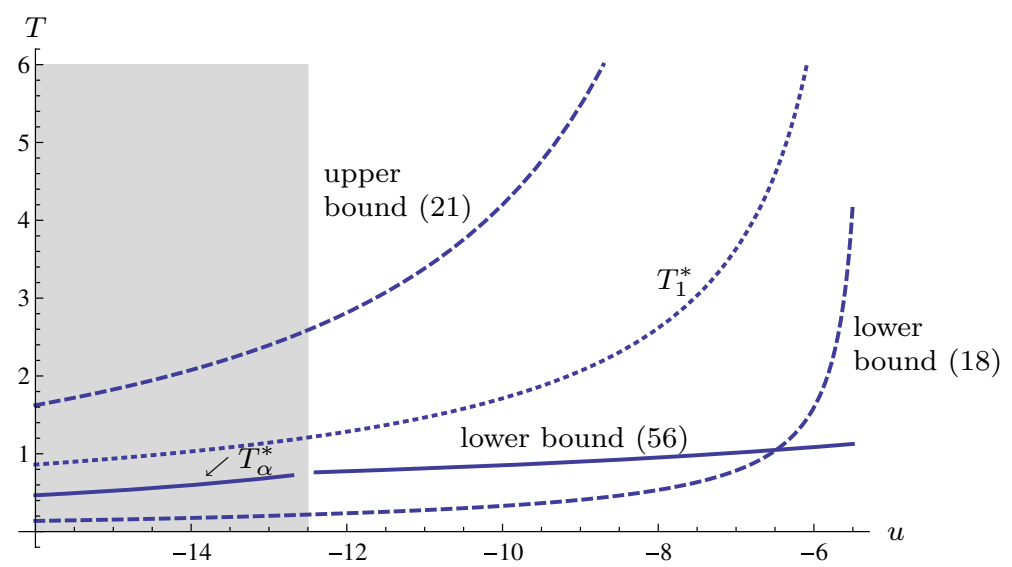

Fig. 2 As Fig. 1, but with $\alpha=0.75$

See Figs. 1, 2 and 3 for numerical examples of these bounds. The following lemma shows that $f$ blows up fast enough for $I^{1-\alpha} f$ to blow up, too. This is important for inferring blowup of the right-hand side of (1); see the remark after the proof.

Lemma 3 In cases $(A)$ and $(B)$, the solution $f=f(u, \cdot)$ of (9) satisfies

$$
\liminf _{t \uparrow \hat{T}_{\alpha}(u)} f(t)\left(\hat{T}_{\alpha}(u)-t\right)^{\alpha}>0 .
$$

Proof Suppose that we are in case (A). We first show

$$
\lim _{t \uparrow \hat{T}_{\alpha}} f(t)\left(\hat{T}_{\alpha}-t\right)^{\alpha} h\left(\hat{T}_{\alpha}-t\right)=\infty,
$$




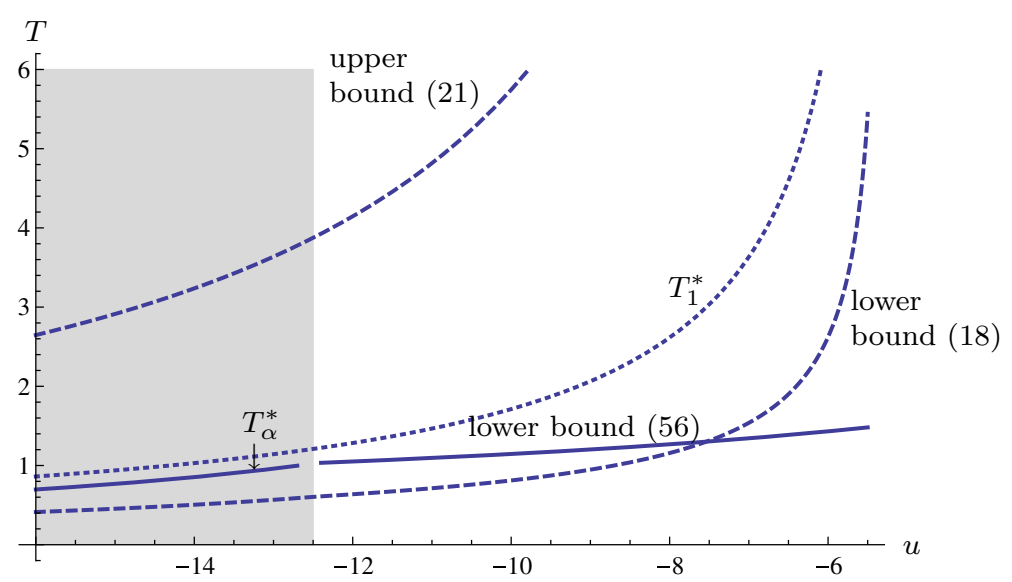

Fig. 3 As Fig. 1, but with $\alpha=0.9$. Note that $T_{\alpha}^{*}$ (left solid curve) is close to $T_{1}^{*}$ (classical Heston model, i.e., $\alpha=1$; dotted curve)

where $h: \mathbb{R}^{+} \rightarrow \mathbb{R}^{+}$is an arbitrary decreasing function satisfying $\lim _{x \downarrow 0} h(x)=\infty$. Fix $r>1$ and $c>0$, and define $t_{n}$ as in the proof of Theorem 4. As in (19), we have

$$
\begin{aligned}
\hat{T}_{\alpha}-t_{n} & =\sum_{k=n+1}^{\infty}\left(t_{k}-t_{k-1}\right) \\
& \geq \Gamma(1+\alpha)^{1 / \alpha} \frac{\left(r^{\alpha}-1\right)^{1 / \alpha}}{r(r-1)} \int_{c r^{n+1}}^{\infty} G\left(s^{\alpha}\right)^{-1 / \alpha} \mathrm{d} s .
\end{aligned}
$$

Since $G\left(s^{\alpha}\right)^{-1 / \alpha}$ is of order $s^{-2}$ at infinity, this implies that there is a constant $C_{1}>0$ (depending on $r$ and $c$ ) such that $\hat{T}_{\alpha}-t_{n} \geq C_{1} r^{-n}$. Assume for contradiction that there is a sequence $0<s_{n} \uparrow \hat{T}_{\alpha}$ with

$$
f\left(s_{n}\right) \leq C_{2}\left(\hat{T}_{\alpha}-s_{n}\right)^{-\alpha} h\left(\hat{T}_{\alpha}-s_{n}\right)^{-1}
$$

for some $C_{2} \geq 0$. Pick sequences $n_{k}, m_{k} \uparrow \infty$ with

$$
t_{n_{k}} \leq s_{m_{k}} \leq t_{n_{k}+1}, \quad k \in \mathbb{N}
$$

In case (A), the function $G$ increases on $[0, \infty)$, and so $f$ increases as well (see, e.g., Theorem 3.1 in Banaś and Martinon (2004)). Therefore,

$$
\begin{aligned}
\left(c r^{n_{k}}\right)^{\alpha} & =f\left(t_{n_{k}}\right) \leq f\left(s_{m_{k}}\right) \\
& \leq C_{2}\left(\hat{T}_{\alpha}-s_{m_{k}}\right)^{-\alpha} h\left(\hat{T}_{\alpha}-s_{m_{k}}\right)^{-1} \\
& \leq C_{2}\left(\hat{T}_{\alpha}-t_{n_{k}+1}\right)^{-\alpha} h\left(\hat{T}_{\alpha}-t_{n_{k}+1}\right)^{-1} \\
& \leq C_{2} C_{1}^{-\alpha} r^{\alpha n_{k}+\alpha} h\left(\hat{T}_{\alpha}-t_{n_{k}+1}\right)^{-1}
\end{aligned}
$$


Since $h(\cdot)^{-1}$ converges to zero by assumption, this upper bound for $\left(c r^{n_{k}}\right)^{\alpha}$ is $o\left(r^{\alpha n_{k}}\right)$ as $k \rightarrow \infty$, which is impossible, and so (24) is established. Now, if (23) were wrong, we could find a sequence $\hat{s}_{n} \uparrow \hat{T}_{\alpha}$ with $\lim _{n \rightarrow \infty} f\left(\hat{s}_{n}\right)\left(\hat{T}_{\alpha}-\hat{s}_{n}\right)^{\alpha}=0$, the convergence being monotone w.l.o.g. This contradicts (24), namely for functions $h$ satisfying

$$
h\left(\hat{T}_{\alpha}-\hat{s}_{n}\right)=\frac{1}{\sqrt{f\left(\hat{s}_{n}\right)\left(\hat{T}_{\alpha}-\hat{s}_{n}\right)^{\alpha}}} .
$$

In case (B), we can show analogously that the function $\bar{f}$ from the proof of Proposition 3 satisfies (23), and conclude by $f \geq \bar{f} \geq 0$.

To obtain blowup of the right-hand side of (1), recall that $f=c_{3} \psi$. By Lemma 3, the blowup of $f$ at $\hat{T}_{\alpha}$ is at least of order $\left(\hat{T}_{\alpha}-t\right)^{-\alpha}$, and so we have a lower bound of order $\left(\hat{T}_{\alpha}-t\right)^{1-2 \alpha}$ for $I^{1-\alpha} f$.

\section{Explosion time in the rough Heston model}

In Sects. 3 and 4, we established that the right-hand side of (1), defined using the solution $f$ of the VIE (9), explodes if and only if $u$ satisfies the conditions of cases (A) or (B). As before, we write $\hat{T}_{\alpha}(u)$ for the explosion time of $f=f(u, \cdot)$. Recall that $T_{\alpha}^{*}(u)$ denotes the explosion time of the rough Heston model, as defined in (11). The goal of the present section is to show that $\hat{T}_{\alpha}(u)=T_{\alpha}^{*}(u)$ and that (1) holds for all $u \in \mathbb{R}$ and $0<t<T_{\alpha}^{*}(u)$. The following result from El Euch and Rosenbaum (2018) was already mentioned at the end of the introduction.

Lemma 4 (Corollary 3.1 in El Euch and Rosenbaum 2018) For each $t>0$, there is an open interval such that (1) holds for all $u$ from that interval.

Lemma 5 The solution $f$ of the Volterra integral equation (9) is differentiable w.r.t. $u$, and its derivative satisfies

$$
\partial_{1} f(u, t)=\int_{0}^{t} \frac{(t-s)^{\alpha-1}}{\Gamma(\alpha)}\left(\partial_{1} G(u, f(u, s))+\partial_{2} G(u, f(u, s)) \partial_{1} f(u, s)\right) \mathrm{d} s .
$$

Proof We check the requirements of Theorem 13.1.2 in Gripenberg et al. (1990).

The polynomial $G(u, w)$ is differentiable. The kernel $(t-s)^{\alpha-1} / \Gamma(\alpha)=: k(t-s)$ is of continuous type in the sense of Gripenberg et al. (1990); see the remark to Theorem 12.1.1 there, which states local integrability of $k$ as a sufficient condition for this property.

Lemma 6 (i) In case (A), we have $\partial_{1} f(u, t)<0$ for $u<0$ and $\partial_{1} f(u, t)>0$ for $u>0$.

(ii) If $u$ satisfies case (B), then the same holds if $\hat{T}_{\alpha}(u)-t$ is sufficiently small.

Proof We only discuss the case $u<0$, because $u>0$ is analogous. 
(i) Note that (25) is a "linear VIE" that can be written as

$$
\partial_{1} f(u, t)=g(t)+\int_{0}^{t}(t-s)^{\alpha-1} K^{(u)}(t, s) \partial_{1} f(u, s) \mathrm{d} s,
$$

where we define

$$
\begin{aligned}
g(t) & :=\int_{0}^{t} \frac{(t-s)^{\alpha-1}}{\Gamma(\alpha)} \partial_{1} G(u, f(u, s)) \mathrm{d} s, \\
K^{(u)}(t, s) & =K^{(u)}(s):=\frac{\partial_{2} G(u, f(u, s))}{\Gamma(\alpha)}
\end{aligned}
$$

to bring the notation close to that of Sect. 6.1.2 in Brunner (2004). Clearly, (26) is not really a linear VIE, because the unknown function $f$ appears in $g$ and $K^{(u)}$. But as our aim is not to solve it, but to control the sign of $\partial_{1} f$, this viewpoint is good enough.

As we are in case (A), we get from $c_{1}(u)>0$ and $e_{0}(u) \geq 0$ that $u \leq \lambda /(\rho \xi)<0$. Furthermore, we have $e_{0}^{\prime}(u)=\xi \rho / 2<0, c_{1}^{\prime}(u)=u-1 / 2<0$ and therefore

$$
f(u, s) e_{0}^{\prime}(u)+\frac{1}{2} c_{3} c_{1}^{\prime}(u)<0,
$$

since $c_{3}=\xi^{2} / 2>0$ and $f(u, s) \geq 0$ by Proposition 2 . From this, we obtain $\partial_{1} G(u, f(u, s))<0$, and hence $g(t)<0$ for all $t \in\left[0, T_{\alpha}^{*}(u)\right)$.

By Theorem 6.1.2 of Brunner (2004), we can express the solution of (25) with the resolvent kernel $R_{\alpha}(\cdot, \cdot)$,

$$
\partial_{1} f(u, t)=g(t)+\int_{0}^{t} R_{\alpha}(t, s) g(s) \mathrm{d} s .
$$

The resolvent kernel has the explicit representation (see Brunner 2004)

$$
R_{\alpha}(t, s)=(t-s)^{\alpha-1} \sum_{n=1}^{\infty} Q_{\alpha, n}(t, s),
$$

where

$$
\begin{aligned}
& Q_{\alpha, 1}(t, s):=K^{(u)}(t, s)=K^{(u)}(s), \\
& Q_{\alpha, n}(t, s):=(t-s)^{(n-1) \alpha} \Phi_{\alpha, n}(t, s), \quad n \geq 2, \\
& \Phi_{\alpha, n}(t, s):=K^{(u)}(s) \int_{0}^{1}(1-z)^{\alpha-1} z^{(n-1) \alpha-1} Q_{\alpha, n-1}(t, s+(t-s) z) d z, \quad n \geq 2 .
\end{aligned}
$$

From this representation of the resolvent kernel, and the fact that (28) is nonnegative in case (A), it is obvious that $R_{\alpha} \geq 0$. Since $g<0$, we thus conclude from (29) that $\partial_{1} f(u, t)<0$ for all $t \in\left[0, \hat{T}_{\alpha}(u)\right)$. 
(ii) Recall that we assume that $u<0$, because $u>0$ is analogous. We have to show that

$$
\tau(u):=\inf \left\{0<t<\hat{T}_{\alpha}(u): \partial_{1} f(u, \cdot)<0 \text { on }\left(t, \hat{T}_{\alpha}(u)\right)\right\}
$$

satisfies $\tau(u)<\hat{T}_{\alpha}(u)$. We use the following facts: $\partial_{1} G(u, w)<0$ for $w$ large, $\partial_{2} G(u, w)>0$ for $w$ large, and $f(u, t)$ explodes as $t \uparrow \hat{T}_{\alpha}(u)$. Thus, $g$ from (27) satisfies

$$
\lim _{t \uparrow \hat{T}_{\alpha}(u)} g(t)=-\infty,
$$

and $K^{(u)}$ satisfies $\lim _{t \uparrow \hat{T}_{\alpha}(u)} K^{(u)}(t)=+\infty$. We can therefore pick $\varepsilon>0$ such that

$$
g(t)<0, K^{(u)}(t)>0 \text { for } \hat{T}_{\alpha}(u)-\varepsilon \leq t<\hat{T}_{\alpha}(u) .
$$

For $z \in[0,1]$ and any $s, t<\hat{T}_{\alpha}(u)$ satisfying $\hat{T}_{\alpha}(u)-\varepsilon \leq s \leq t$, we have

$$
s+(t-s) z \geq s \geq \hat{T}_{\alpha}(u)-\varepsilon .
$$

Using this observation in (31), we see from a straightforward induction proof that

$$
Q_{\alpha, n}(t, s)>0, \quad n \geq 1, \hat{T}_{\alpha}(u)-\varepsilon \leq s \leq t<\hat{T}_{\alpha}(u) .
$$

The same then holds for the resolvent kernel (30),

$$
R_{\alpha}(t, s)>0, \quad \hat{T}_{\alpha}(u)-\varepsilon \leq s \leq t<\hat{T}_{\alpha}(u) .
$$

By (29), we obtain

$$
\partial_{1} f(u, t)=g(t)+\int_{0}^{t-\varepsilon} R_{\alpha}(t, s) g(s) \mathrm{d} s+\int_{t-\varepsilon}^{t} R_{\alpha}(t, s) g(s) \mathrm{d} s .
$$

Now note that

$$
\left|\int_{0}^{t-\varepsilon} R_{\alpha}(t, s) g(s) \mathrm{d} s\right| \ll-\int_{t-\varepsilon}^{t} R_{\alpha}(t, s) g(s) \mathrm{d} s \quad \text { as } t \uparrow \hat{T}_{\alpha}(u),
$$

where the right-hand side is positive. Indeed, (36) follows from (33) and (34), as $g(s)$ on the left-hand side of (36) is $O(1)$. Thus, letting $t \uparrow \hat{T}_{\alpha}(u)$, we find that the negative terms $g(t)+\int_{t-\varepsilon}^{t}$ on the right-hand side of (35) dominate. This completes the proof.

Lemma 7 Let $u \in \mathbb{R}$ and $0<t<\hat{T}_{\alpha}(u)$. Then, $f(\cdot, t)$ is analytic at $u$.

Proof According to Sect. 3.1.1 in Brunner (2017), the solution can be constructed by successive iteration and continuation. We just show that the first iteration step leads to an analytic function, because the finitely many further steps needed to arrive at arbitrary $t<\hat{T}_{\alpha}(u)$ can be dealt with analogously. Define the iterates $f_{0}=0$ and

$$
f_{n+1}(v, s):=\frac{1}{\Gamma(\alpha)} \int_{0}^{s}(s-\tau)^{\alpha-1} G\left(v, f_{n}(v, \tau)\right) d \tau, \quad n \geq 0 .
$$


On a sufficiently small time interval, $f_{n}(v, \cdot)$ converges uniformly to $f(v, \cdot)$, and the solution can then be continued by solving an updated integral equation and so on (see Brunner 2017), until we hit $\hat{T}_{\alpha}(v)$. Now fix $u$ and $t$ as in the statement of the lemma. For a sufficiently small open complex neighborhood $U \ni u$, it is easy to see that $t<\hat{T}_{\alpha}(v)$ holds for $v \in U$. Define

$$
\gamma:=1 \vee \sup _{v \in U}|v| \text { and } \eta:=1 \vee \frac{t^{\alpha}}{\Gamma(\alpha+1)}
$$

Then, there is $c \geq 1$ such that, for arbitrary $v \in U$ and $w \in \mathbb{C}$,

$$
\begin{aligned}
|G(v, w)| & \leq c\left((|w| \vee 1)^{2} \vee \gamma(|w| \vee 1) \vee \gamma^{2}\right) \\
& \leq c \gamma^{2}(|w| \vee 1)^{2}=: \theta(|w| \vee 1)^{2}
\end{aligned}
$$

By the definition of $f_{n}$, a trivial inductive proof then shows that

$$
\sup _{\substack{v \in U \\ s \in[0, t]}}\left|f_{n}(v, s)\right| \leq(\theta \eta)^{2^{n}-1}, \quad n \geq 0 .
$$

By a standard result on parameter integrals (Theorem IV.5.8 in Elstrodt (2009)), bound (37) implies that each function $f_{n}(\cdot, t)$ is analytic in $U$. From the bounds in Sect. 3.1.1 of Brunner (2017), it is very easy to see that the convergence $f_{n}(v, t) \rightarrow$ $f(v, t)$ is locally uniform w.r.t. $v$ for fixed $t$. It is well known [see Theorem 3.5.1 in Greene and Krantz (2006)] that this implies that the limit function $f(\cdot, t)$ is analytic.

Lemma 8 The function $u \mapsto \hat{T}_{\alpha}(u)$ increases for $u \leq 0$ and decreases for $u \geq 1$.

Proof Recall that $\hat{T}_{\alpha}(u)=\infty$ in cases (C) and (D), which include $u \in[0,1]$. For case (A), the assertion follows from part (i) of Lemma 6. So let $u$ satisfy case (B), where again we assume w.l.o.g. that $u<0$. Suppose that $\hat{T}_{\alpha}(\cdot)$ does not increase. Then, we can pick $u_{0}<0$ such that any left neighborhood of $u_{0}$ contains a point $u$ with $\hat{T}_{\alpha}(u)>\hat{T}_{\alpha}\left(u_{0}\right)$. From the continuity of $\partial_{1} f$ (see Lemma 7), part (ii) of Lemma 6 and the continuity of $\tau$ from (32), there are $u_{1}<u_{0}$ satisfying $\hat{T}_{\alpha}\left(u_{1}\right)>\hat{T}_{\alpha}\left(u_{0}\right)$ and $t_{1}<\hat{T}_{\alpha}\left(u_{0}\right)$ such that $\partial_{1} f(u, t)<0$ in the rectangle

$$
\left\{(u, t): u_{1} \leq u \leq u_{0}, t_{1} \leq t<\hat{T}_{\alpha}\left(u_{1}\right)\right\} .
$$

Then, $\lim _{t \uparrow \hat{T}_{\alpha}\left(u_{0}\right)} f\left(u_{0}, t\right)=\infty$ implies that

$$
\lim _{t \uparrow \hat{T}_{\alpha}\left(u_{0}\right)} f\left(u_{1}, t\right)=\infty
$$

because the inequality $\partial_{1} f(u, t)<0$ shows that $f\left(u_{1}, \cdot\right)$ must explode at least as fast as $f\left(u_{0}, \cdot\right)$. But $(38)$ is a contradiction to $\hat{T}_{\alpha}\left(u_{1}\right)>\hat{T}_{\alpha}\left(u_{0}\right)$. 
Lemma 9 Let $u \in \mathbb{R}$ and $0<t<\hat{T}_{\alpha}(u)$. Then, (1) holds, where (as above) $f=c_{3} \psi$ and $f(u, \cdot)$ is the solution of $(9)$.

Proof We assume that $u<0$, as $u \geq 0$ is handled analogously. By Lemma $8, u \mapsto$ $\hat{T}_{\alpha}(u)$ increases. In this proof, we write $M(u, t)$ for the right-hand side of $(1)$, and $\tilde{M}(u, t)=\mathbb{E}\left[\mathrm{e}^{u X_{t}}\right]$ for the mgf. Now fix $u<0$ and $0<t<\hat{T}_{\alpha}(u)$ such that $(u, t)$ has positive distance from the graph of the increasing function $\hat{T}_{\alpha}(\cdot)$. Clearly, it suffices to consider pairs $(u, t)$ with this property. By Lemma 4, there are $v^{-}<v^{+}$such that

$$
M(v, t)=\tilde{M}(v, t), \quad v^{-}<v<v^{+} .
$$

We now show that (39) extends to $u \leq v \leq v^{+}$by analytic continuation. From general results on characteristic functions [Theorems II.5a and II.5b in Widder (1941)], $v \mapsto \tilde{M}(v, t)$ is analytic in a vertical strip $w^{-}<\operatorname{Re}(v)<w^{+}$of the complex plane and has a singularity at $v=w^{-}$. If we suppose that $w^{-}>u$, then Lemma 7 leads to a contradiction: The left-hand side of (39) would then be analytic at $v=w^{-}$and the right-hand side singular. This shows that (39) can be extended to the left up to $u$ by analytic continuation.

In light of Sect. 3 and Lemma 3 from Sect. 4, the following theorem completes the proof of Theorem 3 from Sect. 2.

Theorem 6 Let $u \in \mathbb{R}$. Then, $\hat{T}_{\alpha}(u)=T_{\alpha}^{*}(u)$, and (1) holds for $0<t<T_{\alpha}^{*}(u)$.

Proof By Lemma 9, it only remains to show that $\hat{T}_{\alpha}(u) \geq T_{\alpha}^{*}(u)$. (Obviously, Lemma 9 implies that $\hat{T}_{\alpha}(u) \leq T_{\alpha}^{*}(u)$.) But this is clear from the continuity of the map $t \mapsto \tilde{M}(u, t)=\mathbb{E}\left[\mathrm{e}^{u X_{t}}\right]$ on the interval $\left(0, T_{\alpha}^{*}(u)\right)$. This continuity follows from the continuity of $t \mapsto X_{t}$, Doob's submartingale inequality and dominated convergence.

For later use (Sect. 9), we give the following alternative argument:

Proof (Another proof that $\hat{T}_{\alpha}(u) \geq T_{\alpha}^{*}(u)$ ) Let us suppose that there is $u_{0}$ with $\hat{T}_{\alpha}\left(u_{0}\right)<T_{\alpha}^{*}\left(u_{0}\right)$. From Theorem 4 , it is easy to see that the continuous function $u \mapsto \hat{T}_{\alpha}(u)$ tends to $+\infty$ as $u<0$ approaches the region where $\hat{T}_{\alpha}(u)=\infty$. Thus, there is $u_{1}>u_{0}$ with $\hat{T}_{\alpha}\left(u_{0}\right)<\hat{T}_{\alpha}\left(u_{1}\right)<T_{\alpha}^{*}\left(u_{0}\right)$.

We have seen in Lemma 7 that $u \mapsto f(u, t)$ is analytic for any fixed $t$. But it is also analytic w.r.t. $t$ for fixed $u$ : From Theorem 1 in Lubich (1983), itself based on earlier work by Miller and Feldstein (1971), it follows that $f(u, \cdot)$ is analytic on the whole interval $\left(0, \hat{T}_{\alpha}(u)\right)$. By Hartogs's theorem [Theorem 1.2.5 in Krantz (1992)], we conclude that the bivariate function $f(\cdot, \cdot)$ is continuous. Thus, the blowup of $f\left(u_{1}, \cdot\right)$ at $\hat{T}_{\alpha}\left(u_{1}\right)$ implies that

$$
\lim _{u \downarrow u_{1}} M\left(u, \hat{T}_{\alpha}\left(u_{1}\right)\right)=\infty .
$$

(Again, we write $M$ for the right-hand side of (1) and $\tilde{M}$ for the mgf.) By Lemma 9, $\tilde{M}\left(\cdot, \hat{T}_{\alpha}\left(u_{1}\right)\right)$ also blows up there and thus has a singularity at $u_{1}$. Since $u_{0}<u_{1}$, we conclude from Corollary II.1b in Widder (1941) that $\tilde{M}\left(u_{0}, \hat{T}_{\alpha}\left(u_{1}\right)\right)=\infty$. As $S=\mathrm{e}^{X}$ is a martingale, this implies that $\tilde{M}\left(u_{0}, t\right)=\infty$ for all $t \geq \hat{T}_{\alpha}\left(u_{1}\right)$. In particular, it contradicts $\hat{T}_{\alpha}\left(u_{1}\right)<T_{\alpha}^{*}\left(u_{0}\right)$. 


\section{Validity of the fractional Riccati equation for complex $u$}

Although the focus of this paper is on real $u$, the mgf needs to be evaluated at complex arguments when used for option pricing (see Sect. 10). The following result fully justifies using the fractional Riccati equation (4), respectively, the VIE (9), to do so. As above, we write $T_{\alpha}^{*}(u)$ for the moment explosion time of $S$ and $\hat{T}_{\alpha}(u)$ for the explosion time of the VIE (9).

Theorem 7 Let $u \in \mathbb{C}$. Then, $T_{\alpha}^{*}(u)=T_{\alpha}^{*}(\operatorname{Re}(u))$, and (1) holds for $0<t<T_{\alpha}^{*}(u)$.

Lemma 10 Let $u \in \mathbb{C}$. Then, $\hat{T}_{\alpha}(u) \geq T_{\alpha}^{*}(u)$.

Proof Suppose that $\hat{T}_{\alpha}(u)<T_{\alpha}^{*}(u)$. The VIE (9) translates into a two-dimensional real VIE for $(\operatorname{Re}(f), \operatorname{Im}(f))$. As $\hat{T}_{\alpha}(u)<\infty$, we get from Theorem 12.1.1 in Gripenberg et al. (1990) that $(\operatorname{Re}(f), \operatorname{Im}(f))$ explodes as $t \uparrow \hat{T}_{\alpha}(u)$. This contradicts the continuity of $t \mapsto \mathbb{E}\left[\mathrm{e}^{u X_{t}}\right]$, where the latter is shown as in the proof of Theorem 6 .

Proof of Theorem 7 The first statement is clear from $\left|\mathrm{e}^{u X_{t}}\right|=\mathrm{e}^{\operatorname{Re}(u) X_{t}}$. Now let $t>0$ be arbitrary. As above, we write $\tilde{M}$ for the mgf and $M$ for the right-hand side of (1). By Theorem 6 , we have $M(v, t)=\tilde{M}(v, t)$ for $v$ in the real interval

$$
I:=\left\{v \in \mathbb{R}: T_{\alpha}^{*}(v) \geq t\right\} .
$$

The function $\tilde{M}(\cdot, t)$ is analytic on the strip

$$
\{v \in \mathbb{C}: \operatorname{Re}(v) \in I\}=\left\{v \in \mathbb{C}: T_{\alpha}^{*}(v) \geq t\right\} .
$$

By the same argument as in Lemma 7 , the function $M(\cdot, t)$ is analytic on the set $\left\{v \in \mathbb{C}: \hat{T}_{\alpha}(v) \geq t\right\}$, which contains the strip (41) by Lemma 10 . Therefore, $M(\cdot, t)$ and $\tilde{M}(\cdot, t)$ agree on $(41)$ by analytic continuation. This implies the assertion.

\section{Computing the explosion time}

Recall that, for fixed $u \in \mathbb{R}$, the explosion time $T_{\alpha}^{*}(u)$ of the rough Heston model is the blowup time of $f(t)=f(u, t)=c_{3} \psi(u, t)$, where $\psi$ solves the fractional Riccati initial value problem (4)-(5). We know from Theorem 3 that $T_{\alpha}^{*}(u)<\infty$ exactly in the cases (A) and (B), defined in Sect. 2. We now develop a method (Algorithm 1) to compute $T_{\alpha}^{*}(u)$ for $u$ satisfying the conditions of case (A). In case (B), a lower bound can be computed, which is sometimes sharper than the explicit bound (18). We note that the recent preprint (Callegaro et al. 2019) extends and complements our computational approach. The function $f$ satisfies the fractional Riccati equation

$$
D^{\alpha} f=d_{1}+d_{2} f+f^{2}
$$

where $d_{1}(u):=c_{1}(u) c_{3}$ and $d_{2}(u):=c_{2}(u)$, with initial condition $I^{1-\alpha} f(0)=0$. (Recall that we often suppress the dependence on $u$ in the notation.) We try a fractional 
power series ansatz

$$
f(t) \stackrel{!}{=} \sum_{n=1}^{\infty} a_{n}(u) t^{\alpha n}
$$

with unknown coefficients $a_{n}=a_{n}(u)$.

Lemma 11 (see e.g., Kilbas et al. 2006) Let $\alpha \in(0,1)$. The fractional integral and derivative of power functions are given by

$$
\begin{array}{rlrl}
I_{t}^{\alpha} t^{v} & =t^{\nu+\alpha} \frac{\Gamma(v+1)}{\Gamma(v+\alpha+1)} & \text { for } v>-1, \\
D_{t}^{\alpha} t^{v}=t^{\nu-\alpha} \frac{\Gamma(v+1)}{\Gamma(v-\alpha+1)} & \text { for } v>-1+\alpha .
\end{array}
$$

By (44), the fractional power series (43) (formally) satisfies the initial condition (5). Inserting (43) into (42) and using (45), we obtain

$$
\begin{aligned}
\sum_{n=0}^{\infty} a_{n+1} v_{n+1} t^{\alpha n} & =d_{1}+\sum_{n=1}^{\infty} d_{2} a_{n} t^{\alpha n}+\sum_{n=2}^{\infty}\left(\sum_{k=1}^{n-1} a_{k} a_{n-k}\right) t^{\alpha n} \\
& =d_{1}+d_{2} a_{1} t^{\alpha}+\sum_{n=2}^{\infty}\left(d_{2} a_{n}+\sum_{k=1}^{n-1} a_{k} a_{n-k}\right) t^{\alpha n}
\end{aligned}
$$

where

$$
v_{n}:=\frac{\Gamma(\alpha n+1)}{\Gamma(\alpha n-\alpha+1)} .
$$

Note that $v_{n}$ is an increasing sequence; this follows easily from the fact that $\log \circ \Gamma$ is convex [see Example 11.14 in Schilling (2005)]. By Stirling's formula, $v_{n} \sim(\alpha n)^{\alpha}$ for $n \rightarrow \infty$.

From (46), we obtain a convolution recurrence for $a_{n}=a_{n}(u)$ :

$$
\begin{aligned}
a_{1}(u) & =d_{1}(u) / v_{1}, \\
a_{n+1}(u) & =\frac{1}{v_{n+1}}\left(d_{2}(u) a_{n}(u)+\sum_{k=1}^{n-1} a_{k}(u) a_{n-k}(u)\right), \quad n \geq 1 .
\end{aligned}
$$

The function $f$ can thus be expressed as $f(u, t)=F\left(u, t^{\alpha}\right)$, where

$$
F(u, z):=\sum_{n=1}^{\infty} a_{n}(u) z^{n} .
$$

Lemma 12 Let $u \in \mathbb{R}$, satisfying case $(A)$ or $(B)$ (recall the definition in Sect. 2). Then, $F(u, \cdot)$ is analytic at zero, with a positive and finite radius of convergence $R(u)$. 
Proof To see that the radius of convergence is positive, we show that there is $A=$ $A(u)>0$ such that

$$
\left|a_{n}\right| \leq A^{n} n^{\alpha-1}, \quad n \geq 1 .
$$

(Adding the factor $n^{\alpha-1}$ to this geometric bound facilitates the inductive proof.) We have

$$
\frac{\alpha^{-\alpha}\left|d_{2}\right| n^{-1}}{(n+1)^{\alpha-1}}+\frac{2 \alpha^{-\alpha} \Gamma(\alpha)^{2} n^{\alpha-1}}{\Gamma(2 \alpha)(n+1)^{\alpha-1}} \rightarrow \frac{2 \alpha^{-\alpha} \Gamma(\alpha)^{2}}{\Gamma(2 \alpha)}, \quad n \rightarrow \infty .
$$

Choose $n_{0}$ such that the left-hand side is $\leq 3 \alpha^{-\alpha} \Gamma(\alpha)^{2} / \Gamma(2 \alpha)$ for all $n \geq n_{0}$, and such that $2 v_{n} \geq(\alpha n)^{\alpha}$ for all $n \geq n_{0}$. The latter is possible because $v_{n} \sim(\alpha n)^{\alpha}$. Fix a number $A$ with $A \geq 3 \alpha^{-\alpha} \Gamma(\alpha)^{2} / \Gamma(2 \alpha)$ and such that $A^{n} n^{\alpha-1} \geq\left|a_{n}\right|$ holds for $1 \leq n \leq n_{0}$. Let $n \geq n_{0}$ and assume, inductively, that $\left|a_{k}\right| \leq A^{k} k^{\alpha-1}$ holds for $1 \leq k \leq n$. From the recurrence (48), we then obtain

$$
\begin{aligned}
\left|a_{n+1}\right| & \leq 2(\alpha n+\alpha)^{-\alpha}\left(\left|d_{2}\right| A^{n} n^{\alpha-1}+A^{n} \sum_{k=1}^{n-1} k^{\alpha-1}(n-k)^{\alpha-1}\right) \\
& \leq 2(\alpha n)^{-\alpha}\left(\left|d_{2}\right| A^{n} n^{\alpha-1}+A^{n} \sum_{k=1}^{n-1} k^{\alpha-1}(n-k)^{\alpha-1}\right) .
\end{aligned}
$$

Since $x^{\alpha-1}(n-x)^{\alpha-1}$ is a strictly convex function of $x$ on $(0, n)$ with minimum at $n / 2$, it is easy to see that

$$
\begin{aligned}
\sum_{k=1}^{n-1} k^{\alpha-1}(n-k)^{\alpha-1} & \leq \int_{0}^{n} x^{\alpha-1}(n-x)^{\alpha-1} \mathrm{~d} x \\
& =n^{2 \alpha-1} \int_{0}^{1} y^{\alpha-1}(1-y)^{\alpha-1} \\
& =\frac{\Gamma(\alpha)^{2}}{\Gamma(2 \alpha)} n^{2 \alpha-1}
\end{aligned}
$$

where the last equality follows from the well-known representation of the beta function in terms of the gamma function [see 12.41 in Whittaker and Watson (1996)]. We conclude

$$
\begin{aligned}
\left|a_{n+1}\right| & \leq 2 \alpha^{-\alpha}\left|d_{2}\right| A^{n} n^{-1}+\frac{2 \alpha^{-\alpha} \Gamma(\alpha)^{2}}{\Gamma(2 \alpha)} A^{n} n^{\alpha-1} \\
& =A^{n}(n+1)^{\alpha-1}\left(\frac{2 \alpha^{-\alpha}\left|d_{2}\right| n^{-1}}{(n+1)^{\alpha-1}}+\frac{2 \alpha^{-\alpha} \Gamma(\alpha)^{2} n^{\alpha-1}}{\Gamma(2 \alpha)(n+1)^{\alpha-1}}\right) \\
& \leq A^{n}(n+1)^{\alpha-1} \frac{3 \alpha^{-\alpha} \Gamma(\alpha)^{2}}{\Gamma(2 \alpha)} \\
& \leq A^{n+1}(n+1)^{\alpha-1} .
\end{aligned}
$$

This completes the inductive proof of (49). 
The finiteness of the radius of convergence follows from the fact that $f$ blows up in finite time in cases (A) and (B). In case (A), we can alternatively show in an elementary way that there is a number $B=B(u)>0$ such that

$$
a_{n} \geq B^{n}, \quad n \geq 1
$$

To this end, define

$$
r_{n}:=\frac{d_{2}+n-1}{v_{n+1}}, \quad n \geq 1 .
$$

By Stirling's formula, we have $r_{n} / r_{n-1}=1+(1-\alpha) / n+O\left(n^{-2}\right)$ as $n \rightarrow \infty$, and so $r_{n}$ eventually increases. Let $n_{0} \geq 2$ be such that $r_{n}$ increases for $n \geq n_{0}$, and define

$$
B:=\min \left\{r_{n_{0}}, a_{1}, a_{2}^{1 / 2}, \ldots, a_{n_{0}}^{1 / n_{0}}\right\}
$$

This number satisfies $a_{n} \geq B^{n}$ for $n \leq n_{0}$ by definition. Let us fix some $n \geq n_{0}$ and assume, inductively, that $a_{k} \geq B^{k}$ holds for $1 \leq k \leq n$. By (48)

$$
\begin{aligned}
a_{n+1} & \geq \frac{1}{v_{n+1}}\left(d_{2} B^{n}+(n-1) B^{n}\right) \\
& =B^{n} r_{n} \\
& \geq B^{n} r_{n_{0}} \geq B^{n+1} .
\end{aligned}
$$

Thus, (50) is proved by induction.

From the estimates in Lemma 12, it is clear that termwise fractional derivation of the series (43) is allowed, and so the right-hand side of (43) really represents the solution $f$ of (42) with initial condition $I^{1-\alpha} f(0)=0$, as long as $t$ satisfies $0 \leq t<R(u)^{1 / \alpha}$. We proceed to show how the explosion time $T_{\alpha}^{*}(u)$ can be computed from the coefficients $a_{n}(u)$. The essential fact is that there is no gap between $R(u)^{1 / \alpha}$ and $T_{\alpha}^{*}(u)$. For this, we require the following classical result from complex analysis (Remmert 1991, p. 235).

Theorem 8 (Pringsheim's theorem, 1894) Suppose that the power series $F(z)=$ $\sum_{n=0}^{\infty} a_{n} z^{n}$ has positive finite radius of convergence $R$ and that all the coefficients are nonnegative real numbers. Then, $F$ has a singularity at $R$.

Theorem 9 Suppose that $u \in \mathbb{R}$ satisfies case (A). Define the sequence $a_{n}(u)$ by the recurrence (48) with initial value (47). Then, we have

$$
\limsup _{n \rightarrow \infty} a_{n}(u)^{-1 /(\alpha n)}=T_{\alpha}^{*}(u) .
$$

Proof Recall that $f(u, \cdot)$, the solution of (42), also solves the Volterra integral equation (9). From the references on smoothness cited before (40), it follows that $f(u, \cdot)$ is analytic on the whole interval $\left(0, T_{\alpha}^{*}(u)\right)$. As $f(u, t)$ blows up for $t \uparrow T_{\alpha}^{*}(u)$ 
by Proposition 2 , and $t \mapsto F\left(u, t^{\alpha}\right)$ is analytic on $\left(0, R(u)^{1 / \alpha}\right)$, we must have $R(u)^{1 / \alpha} \leq T_{\alpha}^{*}(u)$.

Assume for contradiction that $R(u)^{1 / \alpha}<T_{\alpha}^{*}(u)$. Then, $f$ is analytic at $R(u)^{1 / \alpha}$. But since $z \mapsto z^{1 / \alpha}$ is analytic at $R(u)>0$, the composition $F(u, z)=f\left(u, z^{1 / \alpha}\right)$ would be analytic at $z=R(u)$ as well, which contradicts Theorem 8 . Therefore,

$$
R(u)^{1 / \alpha}=T_{\alpha}^{*}(u) .
$$

It is well known that the radius of convergence is given by the Cauchy-Hadamard formula (Remmert 1991, p. 111)

$$
R(u)^{-1}=\limsup _{n \rightarrow \infty} a_{n}(u)^{1 / n},
$$

which concludes the proof.

Note that, in case (B), we can argue similarly as in the preceding proof. However, the coefficients $a_{n}$ are no longer positive, and so Pringsheim's theorem is not applicable. Then, the inequality $R(u)^{1 / \alpha} \leq T_{\alpha}^{*}(u)$ need not be an equality. Still, we can compute a lower bound for the explosion time:

$$
\limsup _{n \rightarrow \infty}\left|a_{n}(u)\right|^{-1 /(\alpha n)} \leq T_{\alpha}^{*}(u)
$$

Now assume that we are in case (A) again. We now discuss how to speed up the convergence in (51). Roberts and Olmstead (1996) studied the blowup behavior of solutions of nonlinear Volterra integral equations with (asymptotically) fractional kernel. Their arguments hinge on the asymptotic behavior of the nonlinearity for large argument. In particular, in our situation, with $G(u, w)$ from (10) satisfying $G(u, w) \sim w^{2}$ for $w \rightarrow \infty$, formula (3.2) in Roberts and Olmstead (1996) yields

$$
f(t) \stackrel{(?)}{\sim} \frac{\Gamma(2 \alpha)}{\Gamma(\alpha)}\left(T_{\alpha}^{*}(u)-t\right)^{-\alpha}, \quad t \uparrow T_{\alpha}^{*}(u) ;
$$

cf. also our Lemma 3. We write $\stackrel{(?)}{\sim}$ for two reasons: First, our integral equation (9) does not quite satisfy the technical assumptions in Roberts and Olmstead (1996). Second, not all steps in Roberts and Olmstead (1996) are rigorous. We proceed, heuristically, to infer refined asymptotics of $a_{n}(u)$ from (53). Define

$$
\Phi(z):=\sum_{n=1}^{\infty} a_{n}(u) R(u)^{n} z^{n}
$$

a power series with radius of convergence 1, by the definition of $R(u)$ in Lemma 12 . Its asymptotics for $z \uparrow 1$ can be derived from (53). Recall that the explosion time and the radius of convergence of $F$ are related by $T_{\alpha}^{*}(u)=R(u)^{1 / \alpha}$.

$$
\Phi(z)=f\left((R z)^{1 / \alpha}\right)
$$




$$
\begin{aligned}
& \stackrel{(?)}{\sim} \frac{\Gamma(2 \alpha)}{\Gamma(\alpha)}\left(T_{\alpha}^{*}-(R z)^{1 / \alpha}\right)^{-\alpha} \\
& =\frac{\Gamma(2 \alpha)}{\Gamma(\alpha)} R^{-1}\left(1-z^{1 / \alpha}\right)^{-\alpha} \\
& \sim \frac{\alpha^{\alpha} \Gamma(2 \alpha)}{\Gamma(\alpha)} R^{-1}(1-z)^{-\alpha}, \quad z \uparrow 1 .
\end{aligned}
$$

The method of singularity analysis [see Sect. VI in Flajolet and Sedgewick (2009)] allows to transfer the asymptotics of $\Phi$ to asymptotics of its Taylor coefficients $a_{n} R^{n}$. Sweeping some analytic conditions under the rug, we arrive at

$$
a_{n}(u) R(u)^{n} \stackrel{(?)}{\sim} \frac{\alpha^{\alpha} \Gamma(2 \alpha)}{\Gamma(\alpha)} R^{-1} \frac{n^{\alpha-1}}{\Gamma(\alpha)}, \quad n \rightarrow \infty,
$$

and thus

$$
a_{n}(u) \stackrel{(?)}{\sim} R(u)^{-n-1} n^{\alpha-1} \frac{\alpha^{\alpha} \Gamma(2 \alpha)}{\Gamma(\alpha)^{2}}, \quad n \rightarrow \infty .
$$

Numerical tests confirm (54), and we have little doubt that it is true (in case (A)). Summing up, $T_{\alpha}^{*}(u)$ can be computed by the following algorithm, which converges much faster than the simpler approximation $\lim _{\sup _{n \rightarrow \infty}} a_{n}^{-1 /(\alpha n)}$ :

Algorithm 1 Let $u$ be a real number satisfying case (A).

- Fix $n_{\max } \in \mathbb{N}$ (e.g., $\left.n_{\max }=100\right)$,

- compute $a_{1}(u), \ldots, a_{n_{\max }}(u)$ by the recursion (48),

- compute the approximation

$$
\left.\left(a_{n}(u) n^{1-\alpha} \frac{\Gamma(\alpha)^{2}}{\alpha^{\alpha} \Gamma(2 \alpha)}\right)^{\frac{-1}{\alpha(n+1)}}\right|_{n=n_{\max }} \approx T_{\alpha}^{*}(u)
$$

for the explosion time.

We stress that while the arguments leading to (54) are heuristic, we have rigorously shown in Theorem 9 that $T_{\alpha}^{*}(u)$ is the lim sup of the left-hand side of (55). The heuristic part is that the subexponential factor $n^{1-\alpha} \times$ const improves the relative error of the approximation from $O\left(\frac{\log n}{n}\right)$ to $O\left(\frac{1}{n^{2}}\right)$. Note that our approach to compute the blowup time can of course be extended to more general fractional Riccati equations. Finally, as mentioned above (see (52)), we can compute a lower bound for $T_{\alpha}^{*}(u)$ if it is finite, but $u$ is outside of case (A):

Algorithm 2 Let $u$ be a real number satisfying case (B).

- Fix $n_{\max } \in \mathbb{N}$ (e.g., $\left.n_{\max }=200\right)$,

- compute $a_{1}(u), \ldots, a_{n_{\max }}(u)$ by the recursion (48),

- compute the approximate lower bound

$$
\left.\left|a_{n}(u)\right|^{-1 /(\alpha n)}\right|_{n=n_{\max }} \lesssim T_{\alpha}^{*}(u)
$$

of the explosion time. 
Remark 1 As for the applicability of Algorithm 1, suppose that $\rho<0$ (with analogous comments applying to the less common case $\rho>0$ ). From (7), we have $e_{0}(u) \sim$ $\frac{1}{2} \rho \xi u>0$ for $u \downarrow-\infty$, and so we are in case (A) for large enough $|u|$. More precisely, case (A) corresponds to the interval $u \in(-\infty, \lambda /(\xi \rho)]$. For $u$ from that interval, the explosion time can be computed by Algorithm 1. To the right of $u=\lambda /(\xi \rho)$, there is a (possibly empty) interval corresponding to case (B), where $T_{\alpha}^{*}(u)$ is still finite, but Algorithm 1 cannot be applied. Still, a lower bound can be computed by (52), and we have the bounds from Theorems 4 and 5, which can be easily evaluated numerically. Proceeding further to the right on the $u$-axis, we encounter an interval containing $[0,1]$, on which $T_{\alpha}^{*}(u)=\infty(\operatorname{cases}(\mathrm{C})$ and $(\mathrm{D}))$. Afterward, $T_{\alpha}^{*}(u)$ becomes finite again, but these $u$ belong to case (B), leaving us with bounds for $T_{\alpha}^{*}(u)$ only.

To conclude this section, we note that $f$ can be approximated by replacing the coefficients in (43) by the right-hand side of (54). Let us write $b_{n}(u)$ for the latter. Retaining the first $N$ exact coefficients, this leads to the approximation

$$
\begin{aligned}
f(u, t) & \approx \sum_{n=1}^{\infty} b_{n}(u) t^{\alpha n}+\sum_{n=1}^{N}\left(a_{n}(u)-b_{n}(u)\right) t^{\alpha n} \\
& =\frac{\alpha^{\alpha} \Gamma(2 \alpha)}{\Gamma(\alpha)^{2} T_{\alpha}^{*}(u)^{\alpha}} \operatorname{Li}_{1-\alpha}\left(\left(t / T_{\alpha}^{*}(u)\right)^{\alpha}\right)+\sum_{n=1}^{N}\left(a_{n}(u)-b_{n}(u)\right) t^{\alpha n},
\end{aligned}
$$

where $\operatorname{Li}_{v}(z):=\sum_{n=1}^{\infty} z^{n} / n^{\nu}$ denotes the polylogarithm. While this approximation seems to be very accurate even for small $N$ (see Gerstenecker 2018), it is limited to real $u$ satisfying case (A) and thus not applicable to option pricing (see Sect. 10). Possibly, (57) can be applied in a saddle point approximation of the rough Heston call price for large or small strike; see the end of Sect. 11.

\section{Finiteness of the critical moments}

While we have analyzed the explosion time of the rough Heston model so far, in most applications of moment explosions (see the introduction), the critical moments

$$
\begin{aligned}
& u^{+}(T):=\sup \left\{u \in \mathbb{R}: \mathbb{E}\left[\mathrm{e}^{u X_{T}}\right]<\infty\right\}, \\
& u^{-}(T):=\inf \left\{u \in \mathbb{R}: \mathbb{E}\left[\mathrm{e}^{u X_{T}}\right]<\infty\right\}, \quad T>0,
\end{aligned}
$$

are of interest. Using the upper bound for the moment explosion time $T_{\alpha}^{*}$ in Theorem 5, we will now show the finiteness of the critical moments for every maturity $T>0$. Computing $u^{+}(T)$ and $u^{-}(T)$ is discussed in Sect. 9.

Theorem 10 In the rough Heston model, the critical moments $u^{+}(T)$ and $u^{-}(T)$ are finite for every $T>0$.

Proof Only the finiteness of $u^{+}(T)$ is proven, as the proof for $u^{-}(T)$ is very similar. Denote the upper bound of $T_{\alpha}^{*}(u)$ in (21) by $B(u)$ for all $u \in \mathbb{R}$ in the cases (A) 
and (B). First, we show that for sufficiently large $u$, we are always in case (A) or (B), depending on the sign of the correlation parameter $\rho$. From (7) and (8), it is easy to see that

$$
e_{0}(u) \sim \frac{1}{2} \xi \rho u \text { and } e_{1}(u) \sim-\frac{1}{4} \xi^{2} \bar{\rho}^{2} u^{2} \text { as } u \rightarrow \infty,
$$

where $\bar{\rho}^{2}=1-\rho^{2}$. Thus, $e_{1}(u)<0$ for sufficiently large $u$. In the next step, we show that the upper bound $B(u)$ converges to 0 as $u \rightarrow \infty$. Indeed, in case (A) the integral in (21) satisfies

$$
\begin{aligned}
\int_{0}^{\infty}\left(\frac{w}{G(u, w)}\right)^{1 / \alpha} \frac{\mathrm{d} w}{w} & =\int_{0}^{u}\left(\frac{w}{G(u, w)}\right)^{1 / \alpha} \frac{\mathrm{d} w}{w}+\int_{u}^{\infty}\left(\frac{w}{G(u, w)}\right)^{1 / \alpha} \frac{\mathrm{d} w}{w} \\
& \leq G(u, 0)^{-1 / \alpha} \int_{0}^{u} w^{-1+1 / \alpha} \mathrm{d} w+\int_{u}^{\infty} w^{-1-1 / \alpha} \mathrm{d} w \\
& \leq c u^{-1 / \alpha}, \quad u \rightarrow \infty
\end{aligned}
$$

for some $c>0$, using the monotonicity $G(u, w) \geq G(u, 0)$, the inequality $G(u, w) \geq$ $w^{2}$ and $G(u, 0)=c_{3} c_{1}(u) \sim \frac{1}{2} c_{3} u^{2}$ as $u \rightarrow \infty$.

If we are eventually in case (B) as $u \rightarrow \infty$, then $-e_{1}(u)>0$ and $-e_{0}(u)>0$ holds for all sufficiently large $u$. Note that in this case $G(u, \cdot)$ attains its global minimum at $-e_{0}(u)$ and the minimum value is $-e_{1}(u)$. Thus, the integral in (21) satisfies

$$
\begin{aligned}
& \int_{0}^{\infty}\left(\frac{w}{G(u, w)}\right)^{1 / \alpha} \frac{\mathrm{d} w}{w} \\
& \quad=\int_{0}^{-2 e_{0}(u)}\left(\frac{w}{G(u, w)}\right)^{1 / \alpha} \frac{\mathrm{d} w}{w}+\int_{-2 e_{0}(u)}^{\infty}\left(\frac{w}{G(u, w)}\right)^{1 / \alpha} \frac{\mathrm{d} w}{w} \\
& \quad \leq\left(-e_{1}(u)\right)^{-1 / \alpha} \int_{0}^{-2 e_{0}(u)} w^{-1+1 / \alpha} \mathrm{d} w+4^{1 / \alpha} \int_{-2 e_{0}(u)}^{\infty} w^{-1-1 / \alpha} \mathrm{d} w \\
& \quad=\alpha\left(\left(-e_{1}(u)\right)^{-1 / \alpha}\left(-2 e_{0}(u)\right)^{1 / \alpha}+4^{1 / \alpha}\left(-2 e_{0}(u)\right)^{-1 / \alpha}\right) \\
& \leq c u^{-1 / \alpha}, \quad u \rightarrow \infty,
\end{aligned}
$$

for some $c>0$, using the monotonicity $G(u, w) \geq-e_{1}(u)$, the inequality $G(u, w) \geq$ $\left(w+e_{0}(u)\right)^{2} \geq w^{2} / 4$ on $\left[-2 e_{0}(u), \infty\right)$ and (59).

Altogether, we have $\lim _{u \rightarrow \infty} B(u)=0$. Since $0 \leq T_{\alpha}^{*}(u) \leq B(u)$, the same is true for the moment explosion time $T_{\alpha}^{*}$, i.e., $\lim _{u \rightarrow \infty} T_{\alpha}^{*}(u)=0$. Now let $T>0$ be arbitrary. Then, there exists $u_{0} \in \mathbb{R}$ such that $T_{\alpha}^{*}(u)<T$ for all $u \geq u_{0}$. This inequality implies $\mathbb{E}\left[\mathrm{e}^{u X_{T}}\right]=\infty$ for all $u \geq u_{0}$, and therefore $u_{+}(T) \leq u_{0}$.

From the preceding proof, it easily follows that $u^{+}(T)$ and $u^{-}(T)$ are of order $T^{-\alpha}$ as $T \downarrow 0$. This is consistent with the classical Heston model ( $\alpha=1)$, where the decay order is $T^{-1}$, by inverting (13). 


\section{Computing the critical moments}

We first collect some simple facts that apparently have not been made explicit in the literature on moment explosions. Moment explosion time and critical moments are defined as in (11) resp. (58).

Lemma 13 Let $S=\left(S_{t}\right)_{t \geq 0}=\left(e^{X_{t}}\right)_{t \geq 0}$ be a positive stochastic process. Its moment explosion time is denoted by $T^{*}(u), u \in \mathbb{R}$, and its critical moments by $u^{-}(T)$ and $u^{+}(T), T>0$.

(i) $T^{*}(u)$ increases for $u \leq 0$ and decreases for $u \geq 0$.

(ii) If $S$ is a martingale, then $u^{+}(T)$ decreases, and $u^{-}(T)$ increases.

(iii) Suppose that $S$ is a martingale. If $T^{*}(u)$ decreases strictly on the interval

$$
\mathcal{D}^{+}:=\left\{u \geq 1: T^{*}(u)<\infty\right\}
$$

then $T^{*}=\left(u^{+}\right)^{-1}$ on $\mathcal{D}^{+}$. Analogously, if $T^{*}(u)$ increases strictly on the interval

$$
\mathcal{D}^{-}:=\left\{u \leq 0: T^{*}(u)<\infty\right\}
$$

then $T^{*}=\left(u^{-}\right)^{-1}$ on $\mathcal{D}^{-}$.

Proof (i) As $T^{*}(0)=\infty$, we may assume that $u>0(u<0$ is analogous $)$. The assertion follows from Jensen's inequality, since $x \mapsto x^{v / u}$ is convex for $0<u \leq v$.

(ii) We just consider $u^{+}$. Since $S$ is a martingale, we have $u^{+} \geq 1$. For any number $u \geq 1$ and $0<T \leq T^{\prime}$, we have

$$
\mathbb{E}\left[S_{T^{\prime}}^{u}\right]=\mathbb{E}\left[\mathbb{E}\left[S_{T^{\prime}}^{u} \mid \mathcal{F}_{T}\right]\right] \geq \mathbb{E}\left[S_{T}^{u}\right]
$$

by the conditional Jensen inequality. This shows the assertion.

(iii) We just prove the first statement. Note that (i) implies that $\mathcal{D}^{+}$is an interval. Now suppose for contradiction that $u \in \mathcal{D}^{+}$satisfies

$$
u<u^{+}\left(T^{*}(u)\right)=\sup \left\{v: \mathbb{E}\left[S_{T^{*}(u)}^{v}\right]<\infty\right\}
$$

This means that there is $v>u$ satisfying $\mathbb{E}\left[S_{T^{*}(u)}^{v}\right]<\infty$. Hence, $T^{*}(v)=$ $T^{*}(u)$, contradicting the strict decrease of $T^{*}$. Finally, suppose for contradiction that $u \geq 1$ satisfies $u>u^{+}\left(T^{*}(u)\right)$. Then, there is $1 \leq v<u$ such that $\mathbb{E}\left[S_{T^{*}(u)}^{v}\right]=\infty$. For arbitrary $t \geq T^{*}(u)$, we get

$$
\mathbb{E}\left[S_{t}^{v}\right]=\mathbb{E}\left[\mathbb{E}\left[S_{t}^{v} \mid \mathcal{F}_{T^{*}(u)}\right]\right] \geq \mathbb{E}\left[S_{T^{*}(u)}^{v}\right]=\infty
$$

This implies $T^{*}(v)=T^{*}(u)$, again contradicting the strict decrease of $T^{*}$.

If the assumptions of part (iii) hold, then we can compute the critical moments from the explosion time, by numerically solving the equations $T^{*}\left(u^{+}(T)\right)=T$ resp. $T^{*}\left(u^{-}(T)\right)=T$. Note, however, that strict monotonicity may fail for reasonable 
stochastic volatility models. In the 3/2-model (Lewis 2000), the explicit characteristic function shows that the critical moments do not depend on maturity (for positive maturity), and the explosion time assumes only the values zero and infinity.

In the classical Heston model, on the other hand, it easily follows from (13) that $T_{1}^{*}(u)$ (recall that the index denotes $\alpha=1$ ) is a strictly monotonic function of $u$ : On the set where it is finite, $T_{1}^{*}$ strictly increases for negative $u$ and strictly decreases for positive $u$. By part (iii) of Lemma 13, this implies that we have

$$
T_{1}^{*}\left(u^{+}(T)\right)=T \text { and } T_{1}^{*}\left(u^{-}(T)\right)=T, \quad T>0 .
$$

Thus, although the critical moments do not admit an explicit expression, they can be computed using (13) and (60), by numerical root finding with an appropriate starting value.

While we have no doubt that strict monotonicity of the explosion time extends to the rough Heston model, this seems not easy to verify. If we accept it as given, then the lower critical moment can be computed for $\rho<0$ as the unique solution of

$$
T_{\alpha}^{*}\left(u^{-}(T)\right)=T
$$

Again, we focus on the lower critical moment, because then we can apply Algorithm 1 to compute $T_{\alpha}^{*}$ for $\rho<0$. Recall that this algorithm works only for $u \leq \lambda /(\rho \xi)$, which amounts to case (A). Thus, $T$ must not be too large in (9), namely such that $u^{-}(T)$ satisfies case (A). (Usually, this requirement is not too prohibitive.)

To provide some indication for the strict monotonicity of $T_{\alpha}^{*}$, recall that according to Lemma 6, $f(u, t)=c_{3} \psi(u, t)$ (see Sect. 2) strictly decreases w.r.t. $u$, if $u<0$ satisfies case (A). It is then plausible (although not proven) that the strictly smaller function $f\left(u_{2}, \cdot\right)$ explodes at a larger time than $f\left(u_{1}, \cdot\right)$, where $u_{1}<u_{2}<0$. As another indication, the bounds in Theorems 4 and 5 are strictly monotonous, as seen by differentiating them w.r.t. $u$.

Even in case strict monotonicity should not hold, we certainly have

$$
\begin{aligned}
& u^{-}(T)=\sup \left\{u<0: T_{\alpha}^{*}(u)=T\right\} \\
& u^{+}(T)=\inf \left\{u>1: T_{\alpha}^{*}(u)=T\right\}
\end{aligned}
$$

for all $T>0$, which suffices for numerical computations (under the above restriction on $T$ ). The validity of (61) and (62) is clear from (40): If $T_{\alpha}^{*}(\cdot)$ is constant on some interval, lying to the left of zero, say, then the mgf blows up as $u$ approaches the interval's right endpoint from the right.

\section{Option pricing}

In the introduction we mentioned several potential applications of our work. In this section, we give some details on one of them. W.l.o.g. we assume zero interest rate and $S_{0}=1$. For an arbitrary parameter $1<\beta<u^{+}(T)$, the call price with strike $K$ and maturity $T$ can be computed by the Fourier integral 


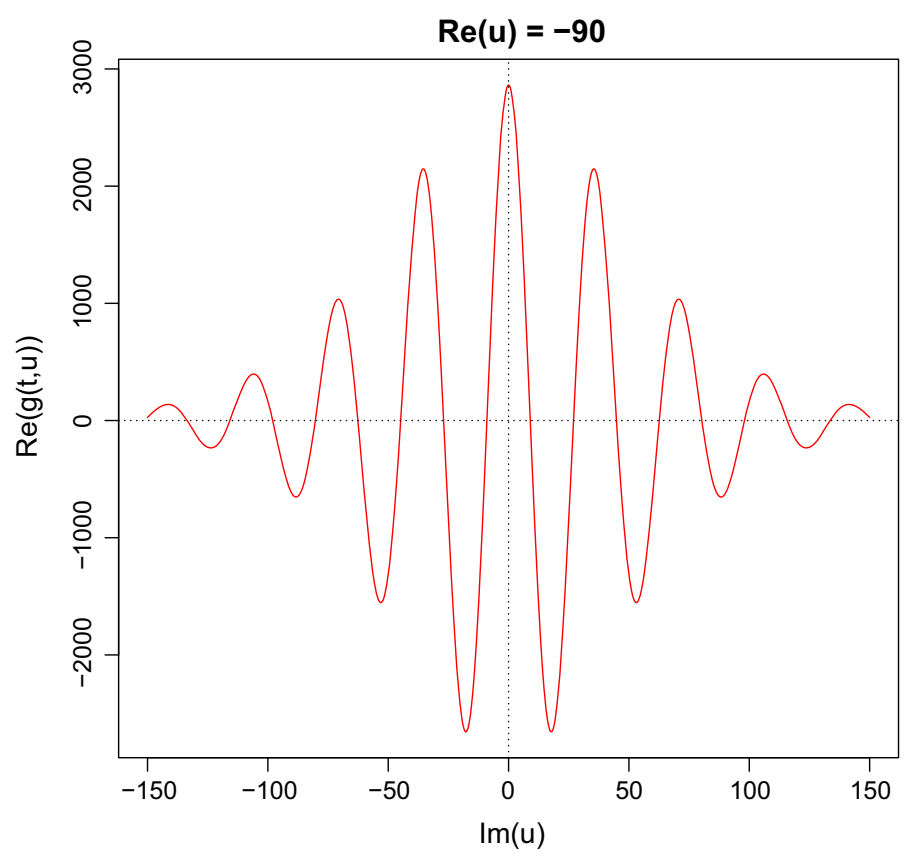

Fig. 4 We plot the real part of $g(t, u):=\mathrm{e}^{-k u} \mathbb{E}\left[\exp \left(u X_{t}\right)\right] /(u(u-1))$ as a function of $\operatorname{Im}(u)$, with $\beta=\operatorname{Re}(u)=-90$ fixed. The roughness parameter is $\alpha=0.6$, and strike has been fixed as $K=\mathrm{e}^{k}=1.2$, with $S_{0}=1$. Recall that only the real part is needed for pricing, as the imaginary part is an odd function whose integral vanishes

$$
\mathbb{E}\left[\left(S_{T}-K\right)^{+}\right]=\frac{1}{2 \pi i} \int_{\beta-i \infty}^{\beta+i \infty} \mathrm{e}^{k(1-u)} \frac{\mathbb{E}\left[\mathrm{e}^{u X_{T}}\right]}{u(u-1)} \mathrm{d} u
$$

See Lee (2004b) and the references therein for more information. The integrand has residues $-K$ at $u=0$ and 1 at $u=1$. Thus, by the residue theorem, the following well-known formula holds for negative $\beta$ :

$$
\mathbb{E}\left[\left(S_{T}-K\right)^{+}\right]+K-1=\frac{1}{2 \pi i} \int_{\beta-i \infty}^{\beta+i \infty} \mathrm{e}^{k(1-u)} \frac{\mathbb{E}\left[\mathrm{e}^{u X_{T}}\right]}{u(u-1)} \mathrm{d} u, \quad u^{-}(T)<\beta<0
$$

Even for Markovian stochastic volatility models, it is well known that the choice of the parameter $\beta$ greatly affects the performance of numerical quadrature algorithms when computing call prices in this way. See Lord and Kahl (2007) for a detailed study. For the rough Heston model, this is even more important, because evaluating the mgf is far more costly than in the classical case. A bad choice of $\beta$ can lead to unnecessary oscillations of the integrand, which require a large number of grid points. Moreover, $\beta<u^{-}(T)$ might lead to a numerical result that is not the correct option price, because then the Fourier representation of the call is no longer valid. Our results allow to compute the range of possible values of $\beta$ (with the caveats described in the preceding section). For $\rho<0$, we recommend to use (63) to compute the call price, 


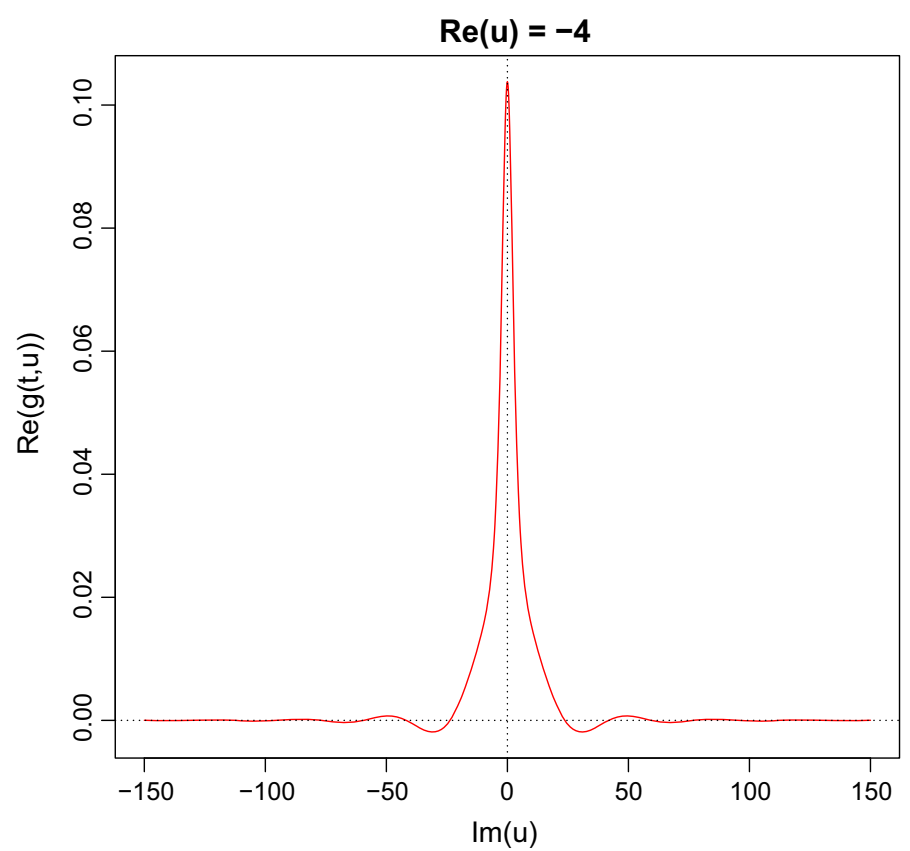

Fig. 5 As Fig. 4, but with $\beta=\operatorname{Re}(u)=-4$. The reduced oscillation greatly simplifies numerical integration of $g$. Note that the oscillations become worse again if $\beta<0$ is increased further

because our Algorithm 1 allows to compute $u^{-}(T)$. Figures 4 and 5 show how the amount of oscillations is affected by the choice of $\beta=\operatorname{Re}(u)$.

\section{Application to asymptotics}

Knowing the critical moments gives first-order asymptotics for the implied volatility for large and small strikes. We write $\hat{\sigma}(k)$ for the implied volatility, where $k=\log \left(K / S_{0}\right)$ is the $\log$-moneyness. According to Lee's moment formula (Lee 2004a), the left wing of implied volatility satisfies

$$
T \cdot \limsup _{k \rightarrow-\infty} \frac{\hat{\sigma}(k)^{2}}{|k|}=2-4\left(\sqrt{u^{-}(T)^{2}-u^{-}(T)}+u^{-}(T)\right) \text {. }
$$

We focus on negative log-moneyness, because then the slope depends on the lower critical moment, which Algorithm 1 computes in the important case $\rho<0$, if $T$ is not too large.

As in any model with finite critical moments, the marginal densities of the rough Heston model have power-law tails. More precisely, if we write $f_{T}$ for the density of $S_{T}$, then

$$
f_{T}(x)=x^{-u^{+}(T)-1+o(1)}, \quad x \rightarrow \infty,
$$

and 


$$
f_{T}(x)=x^{-u^{-}(T)-1+o(1)}, \quad x \downarrow 0 .
$$

Our approach (see Sect. 9) allows to evaluate the right-hand sides of (64) and (66) numerically for the rough Heston model, if $T$ is not too large.

In Friz et al. (2011), (64)-(66) were considerably sharpened for the classical Heston model. We expect that such a refined smile expansion can be done for rough Heston, too, with density asymptotics of the form

$$
f_{T}(x) \sim c_{1} x^{-u^{+}(T)-1} \mathrm{e}^{c_{2}(\log x)^{1-1 /(2 \alpha)}}(\log x)^{c_{3}}, \quad x \rightarrow \infty,
$$

where the $c_{i}$ depend on $T$ and $\alpha$. In the classical Heston model, the factor $\mathrm{e}^{c_{2}(\log x)^{1-1 /(2 \alpha)}}$ becomes $\mathrm{e}^{c_{2} \sqrt{\log x}}$, in line with Friz et al. (2011). From previous studies on simpler models, it is well known that Lee's formula (64) only becomes accurate for very large values of $|k|$. Therefore, a refined analysis of the wings is necessary to obtain an asymptotic formula of practical use. Extending the analysis of Friz et al. (2011) to $\frac{1}{2}<\alpha<1$ will require a detailed study of the blowup behavior of the Volterra integral equation (9). Among other things, (a special case of) the heuristic analysis in Roberts and Olmstead (1996), which we already mentioned in Sect. 7, would have to be made rigorous and extended to ensure uniformity w.r.t. the parameter $u$. We postpone this to future work. Note that the approximation (57) might be useful in this context.

Acknowledgements Open access funding provided by Austrian Science Fund (FWF). We thank Omar El Euch, Paul Gassiat, Antoine Jacquier and Martin Keller-Ressel for helpful comments.

Open Access This article is distributed under the terms of the Creative Commons Attribution 4.0 International License (http://creativecommons.org/licenses/by/4.0/), which permits unrestricted use, distribution, and reproduction in any medium, provided you give appropriate credit to the original author(s) and the source, provide a link to the Creative Commons license, and indicate if changes were made.

\section{References}

Abi Jaber, E., Larsson, M., Pulido, S.: Affine Volterra processes. Preprint arXiv:1708.08796 (2017)

Alòs, E., León, J.A., Vives, J.: On the short-time behavior of the implied volatility for jump-diffusion models with stochastic volatility. Finance Stoch. 11(4), 571-589 (2007)

Alòs, E., Gatheral, J., Radoicic, R.: Exponentiation of conditional expectations under stochastic volatility. Preprint (2017). https://ssrn.com/abstract=2983180

Andersen, L.B.G., Piterbarg, V.V.: Moment explosions in stochastic volatility models. Finance Stoch. 11(1), 29-50 (2007)

Banaś, J., Martinon, A.: Monotonic solutions of a quadratic integral equation of Volterra type. Comput. Math. Appl. 47(2-3), 271-279 (2004)

Bayer, C., Friz, P.K., Gassiat, P., Martin, J., Stemper, B.: A regularity structure for rough volatility. Preprint arXiv:1710.07481 (2017)

Brunner, H.: Collocation methods for Volterra integral and related functional differential equations. In: Ciarlet, P.G., et al. (eds.) Cambridge Monographs on Applied and Computational Mathematics, vol. 15. Cambridge University Press, Cambridge (2004)

Brunner, H.: Volterra Integral Equations, Cambridge Monographs on Applied and Computational Mathematics, vol. 30. Cambridge University Press, Cambridge (2017)

Brunner, H., Yang, Z.W.: Blow-up behavior of Hammerstein-type Volterra integral equations. J. Integral Equ. Appl. 24(4), 487-512 (2012)

Callegaro, G., Grasselli, M., Pagès, G.: Fast hybrid schemes for fractional Riccati equations (rough is not so tough). Preprint arXiv:1805.12587 (2019) 
Comte, F., Renault, E.: Long memory in continuous-time stochastic volatility models. Math. Finance 8(4), 291-323 (1998)

Comte, F., Coutin, L., Renault, E.: Affine fractional stochastic volatility models. Ann. Finance 8(2-3), 337-378 (2012)

Cont, R.: Encyclopedia of Quantitative Finance. Wiley, Berlin (2014)

El Euch, O., Rosenbaum, M.: Perfect hedging in rough Heston models. Ann. Appl. Probab. 28(6), 38133856 (2018)

El Euch, O., Rosenbaum, M.: The characteristic function of rough Heston models. Math. Finance 29(1), 3-38 (2019)

Elstrodt, J.: Maß- und Integrationstheorie. Springer-Lehrbuch, 6th edn. Springer, Berlin (2009)

Flajolet, P., Sedgewick, R.: Analytic Combinatorics. Cambridge University Press, Cambridge (2009)

Friz, P., Gerhold, S., Gulisashvili, A., Sturm, S.: On refined volatility smile expansion in the Heston model. Quant. Finance 11(8), 1151-1164 (2011)

Fukasawa, M.: Asymptotic analysis for stochastic volatility: martingale expansion. Finance Stoch. 15(4), 635-654 (2011)

Gatheral, J.: The Volatility Surface, a Practitioner's Guide. Wiley, Berlin (2006)

Gatheral, J., Keller-Ressel, M.: Affine forward variance models. Finance Stoch 23(3), 501-533 (2019)

Gatheral, J., Jaisson, T., Rosenbaum, M.: Volatility is rough. Quant. Finance 18(6), 933-949 (2018)

Gerstenecker, C.: Moment explosion time in the rough Heston model. Master's Thesis, TU Wien (2018)

Greene, R.E., Krantz, S.G.: Function theory of one complex variable. In: In: Krantz, S.G., et al. (eds.) Graduate Studies in Mathematics, vol. 40, 3rd edn. American Mathematical Society, Providence (2006)

Gripenberg, G., Londen, S.O., Staffans, O.: Volterra Integral and Functional Equations, Encyclopedia of Mathematics and its Applications, vol. 34. Cambridge University Press, Cambridge (1990)

Keller-Ressel, M.: Moment explosions and long-term behavior of affine stochastic volatility models. Math. Finance 21(1), 73-98 (2011)

Keller-Ressel, M., Majid, A.: A comparison principle between rough and non-rough Heston models-with applications to the volatility surface. Preprint arXiv:1906.03119 (2019)

Kilbas, A.A., Srivastava, H.M., Trujillo, J.J.: Theory and Applications of Fractional Differential Equations, North-Holland Mathematics Studies, vol. 204. Elsevier Science B.V., Amsterdam (2006)

Krantz, S.G.: Function Theory of Several Complex Variables, 2nd edn. Wadsworth \& Brooks/Cole Advanced Books \& Software, Pacific Grove (1992)

Lee, R.W.: The moment formula for implied volatility at extreme strikes. Math. Finance 14(3), 469-480 (2004a)

Lee, R.W.: Option pricing by transform methods: extensions, unification, and error control. J. Comput. Finance 7(3), 51-86 (2004b)

Lewis, A.L.: Option Valuation Under Stochastic Volatility. Finance Press, Newport Beach (2000)

Lord, R., Kahl, C.: Optimal Fourier inversion in semi-analytical option pricing. Tinbergen Institute Discussion Papers 06-066/2, Tinbergen Institute (2007)

Lubich, C.: Runge-Kutta theory for Volterra and Abel integral equations of the second kind. Math. Comput. 41(163), 87-102 (1983)

Miller, R.K., Feldstein, A.: Smoothness of solutions of Volterra integral equations with weakly singular kernels. SIAM J. Math. Anal. 2, 242-258 (1971)

Remmert, R.: Theory of Complex Functions, Graduate Texts in Mathematics, vol. 122. Springer, New York (1991)

Roberts, C.A.: Analysis of explosion for nonlinear Volterra equations. J. Comput. Appl. Math. 97(1-2), 153-166 (1998)

Roberts, C.A., Olmstead, W.E.: Growth rates for blow-up solutions of nonlinear Volterra equations. Quart. Appl. Math. 54(1), 153-159 (1996)

Schilling, R.L.: Measures, Integrals and Martingales. Cambridge University Press, New York (2005)

Whittaker, E.T., Watson, G.N.: A course of modern analysis. Cambridge Mathematical Library, Cambridge University Press, Cambridge (1996). Reprint of the fourth (1927) edition

Widder, D.: The Laplace Transform. Princeton Mathematical Series. Princeton University Press, Princeton (1941)

Publisher's Note Springer Nature remains neutral with regard to jurisdictional claims in published maps and institutional affiliations. 